\title{
A-PRIORI ANALYSIS AND THE FINITE ELEMENT METHOD FOR A CLASS OF DEGENERATE ELLIPTIC EQUATIONS
}

\author{
HENGGUANG LI
}

\begin{abstract}
Consider the degenerate elliptic operator $\mathcal{L}_{\delta}:=-\partial_{x}^{2}-\frac{\delta^{2}}{x^{2}} \partial_{y}^{2}$ on $\Omega:=(0,1) \times(0, l)$, for $\delta>0, l>0$. We prove well-posedness and regularity results for the degenerate elliptic equation $\mathcal{L}_{\delta} u=f$ in $\Omega,\left.u\right|_{\partial \Omega}=0$ using weighted Sobolev spaces $\mathcal{K}_{a}^{m}$. In particular, by a proper choice of the parameters in the weighted Sobolev spaces $\mathcal{K}_{a}^{m}$, we establish the existence and uniqueness of the solution. In addition, we show that there is no loss of $\mathcal{K}_{a}^{m}$-regularity for the solution of the equation. We then provide an explicit construction of a sequence of finite dimensional subspaces $V_{n}$ for the finite element method, such that the optimal convergence rate is attained for the finite element solution $u_{n} \in V_{n}$, i.e., $\left\|u-u_{n}\right\|_{H^{1}(\Omega)} \leq C \operatorname{dim}\left(V_{n}\right)^{-\frac{m}{2}}\|f\|_{H^{m-1}(\Omega)}$ with $C$ independent of $f$ and $n$.
\end{abstract}

\section{INTRODUCTION}

Let $\Omega$ be the rectangular domain $(0,1) \times(0, l)$ for $l>0$, and define the operator $\mathcal{L}_{\delta}$ as follows:

$$
\mathcal{L}_{\delta}:=-\partial_{x}^{2}-\frac{\delta^{2}}{x^{2}} \partial_{y}^{2}, \quad \delta>0
$$

We then consider a class of degenerate elliptic equations on $\Omega$ corresponding to $\mathcal{L}_{\delta}$ with the Dirichlet boundary condition

$$
\left\{\begin{aligned}
\mathcal{L}_{\delta} u=f & \text { in } \quad \Omega \\
u=0 & \text { on } \quad \partial \Omega
\end{aligned}\right.
$$

Equations of this type often appear in fluid dynamics, especially in mathematical models of fuel cells [32, 48. Therefore, the mathematical study on equation (1) is of practical importance.

Denote by $u$ the solution of equation (11). Let $\Omega_{\xi}:=(0, \xi) \times(0, l), 0<\xi<1$, and $S_{\xi}:=\Omega \backslash \Omega_{\xi}$ be subsets of $\Omega$, depending on $\xi$. Denote by $P_{r} \subset \Omega$, an arbitrary open subset containing the neighborhoods of the vertices $(1,0)$ and $(1, l)$. Then, the strong ellipticity of the operator $\mathcal{L}_{\delta}$ on $S_{\xi} \backslash P_{r}$ implies that there exists a unique solution $u \in H^{m+1}\left(S_{\xi} \backslash P_{r}\right) \cap\left\{\left.u\right|_{\partial \Omega}=0\right\}$ for any $f \in H^{m-1}(\Omega)$ [26, 46].

However, it is well known that this result does not extend to the entire domain $\Omega$ in general, because of the loss of ellipticity at $x=0$ and the possible singularities of the solution arising from the corners. In fact, it is generally impossible to find a solution $u \in H^{m+1}(\Omega)$ for large $m$, even if the given data is

Received by the editor October 18, 2006 and, in revised form, May 2, 2008.

2000 Mathematics Subject Classification. Primary 35J70, 41A25, 41A50, 65N12, 65N15, $65 \mathrm{~N} 30,65 \mathrm{~N} 50$.

H. Li was supported in part by NSF Grant DMS 0713743. 
smooth $f \in \mathcal{C}^{\infty}(\Omega)$. Various techniques have been used to investigate different degenerate elliptic equations, usually depending on the character of the degeneracy. For example, see Boimatov [17, Felli and Schneider [27, French [28], Langlais 37, and references therein. In particular, Gopalakrishnan and Pasciak [29] studied the multigrid method for the axisymmetric Laplace and Maxwell equations, in which singular coefficients of a different kind were considered; Bramble and Zhang 18. estimated the multigrid method for a similar anisotropic equation, based on a regularity result in a weighted $L^{2}$ space, and on the corresponding finite element approximation property for a special "energy norm" by assuming the existence of a global $H^{2}$ solution.

In this paper, inspired by the methods of Babuska and Aziz 9], Bacuta, Nistor, and Zikatanov [15, 16, and Kondratiev [34, we shall study the well-posedness and regularity of the solution of equation (1) in the following weighted Sobolev space $\mathcal{K}_{a}^{m}(\Omega)$. Let $\rho$ be a smooth function, such that $\rho=$ the distance to the set $\{(1,0),(1, l),[0, y]$ for $0 \leq y \leq l\}$ in the neighborhood of it. Also, we denote by $r_{1}$ and $r_{2}$ the distance functions to $(1,0)$ and $(1, l)$, respectively. Then, we define

$$
\mathcal{K}_{a}^{m}(\Omega):=\left\{v \in L_{l o c}^{2}(\Omega), \rho^{-a}\left(r_{1} r_{2}\right)^{i+j}\left(x \partial_{x}\right)^{i} \partial_{y}^{j} v \in L^{2}(\Omega), i+j \leq m\right\} .
$$

See Definition 2.1 for more discussions. The well-posedness of the solution $u$ in these spaces will be proved, and consequently, we shall show that there is no loss of $\mathcal{K}_{a}^{m}$-regularity for the solution of equation (11).

Another main result of this paper is regarding the numerical approximation by the finite element method (FEM). Let $V_{n}$ be a sequence of finite dimensional subspaces for the FEM. Denote by $u_{n} \in V_{n}$ the corresponding discrete solution. Then, we shall provide a simple, explicit way to construct a sequence of finite dimensional subspaces $V_{n} \subset H_{0}^{1}(\Omega)$, such that $u_{n}$ satisfies

$$
\left\|u-u_{n}\right\|_{H^{1}(\Omega)} \leq C \operatorname{dim}\left(V_{n}\right)^{-m / 2}\|f\|_{H^{m-1}(\Omega)},
$$

where $f \in H^{m-1}(\Omega)$ is arbitrary and $C$ is a constant that depends on $\Omega$ and $m$, but not $n$ or $f$. Namely, one can recover the optimal rate of convergence that is expected for smooth solutions [9, 19, 21]. Comparing with the regularity and approximation estimates in [18], here we provide regularity results in weighted Sobolev space of order $m, m \geq 1$, with least assumptions on $u$ and $f$. Besides producing numerical solutions with high-order rates of convergence, our construction of the special finite dimensional subspaces is suitable for any $f \in L^{2}(\Omega)$, in which case, $u \notin H^{2}(\Omega)$ in general.

In addition, according to the result of Babuska and Aziz [10, the maximum angle of the triangles in the triangulation for the FEM should be bounded away from $\pi$, such that a uniform error estimate can be obtained in the usual Sobolev spaces $H^{m}$ on each triangle. Otherwise, the energy norm of the error $\left|u-u_{n}\right|_{H^{1}}$ on $\Omega$ might be difficult to control. In our construction of subspaces $V_{n}$, however, thin triangles that violate this maximum-angle condition will appear. In fact, the maximum angle in the triangles will keep increasing with $\pi$ as the limit. We shall show that the difficulty for the estimates in this case can be overcome by a homogeneity argument in weighted Sobolev spaces.

In Section 2, we shall introduce our weighted Sobolev spaces $\mathcal{K}_{a}^{m}(\Omega)$ and some notation that will be used throughout this paper. The properties of $\mathcal{K}_{a}^{m}(\Omega)$ that are important for the regularity of the solution will be studied in detail. 
In Section 3 we shall prove the well-posedness and regularity of the solution in $\mathcal{K}_{a}^{m}(\Omega)$. Denote by $\left(v_{1}, v_{2}\right)$ the $L^{2}$ inner product of $v_{1}, v_{2} \in H^{0}(\Omega)=L^{2}(\Omega)$. The corresponding weak solution $u \in \mathcal{K}_{1}^{1}(\Omega) \cap\left\{\left.u\right|_{\partial \Omega}=0\right\}$ of equation (1) is defined by

$$
a(u, v):=\left(\partial_{x} u, \partial_{x} v\right)+\delta^{2}\left(\frac{1}{x} \partial_{y} u, \frac{1}{x} \partial_{y} v\right)=\langle f, v\rangle, \quad \forall v \in \mathcal{K}_{1}^{1}(\Omega) \cap\left\{\left.v\right|_{\partial \Omega}=0\right\} .
$$

Furthermore, we prove that, for $m \geq 0$, there exists a constant $\eta>0$, such that

$$
\mathcal{L}_{\delta}=-\partial_{x}^{2}-\frac{\delta^{2}}{x^{2}} \partial_{y}^{2}: \mathcal{K}_{1+\epsilon}^{m+1}(\Omega) \cap\left\{\left.u\right|_{\partial \Omega}=0\right\} \rightarrow \mathcal{K}_{-1+\epsilon}^{m-1}(\Omega), \quad \forall|\epsilon|<\eta,
$$

defines an isomorphism, while it is Fredholm iff $\epsilon$ is away from a specific countable set of values.

In Section 4 we will analyze the numerical solution $u_{n}$ for equation (11). Explicitly, we will look for $u_{n} \in V_{n}$ satisfying

$$
a\left(u_{n}, v_{n}\right)=\left(f, v_{n}\right), \quad \forall v_{n} \in V_{n} .
$$

To better explain our results, let us recall some basic results in classical approximation theory [19, 21, 24]. Denote by $\mathcal{T}=\left\{T_{i}\right\}$ the triangulation of $\Omega$ with triangles. Let $V=V(\mathcal{T}, m+1)$ be the finite element space associated to the degree $m$ Lagrange triangle 21, such that $V$ consists of polynomials of degree $\leq m$ on each triangle $T_{i} \in \mathcal{T}$. Let $u_{V} \in V$ be the finite element solution of equation (11). For any continuous solution $u$, denote by $u_{I} \in V(\mathcal{T}, m+1)$ the nodal interpolation associated to $u$, which is uniquely determined by the condition $u\left(x_{i}\right)=u_{I}\left(x_{i}\right)$ for any node. Also, a symmetric bilinear form $a(\cdot, \cdot)$ induces an equivalent norm $\|\cdot\|_{a}$ on a normed space, provided that $a(\cdot, \cdot)$ is both continuous and coercive on this space. As a result from Section 3 we shall show that $\|\cdot\|_{\mathcal{K}_{1}^{1}}$ and $\|\cdot\|_{a}$ are equivalent norms on $\Omega$. Therefore, based on Céa's Lemma [19, we have the following inequality:

$$
\left\|u-u_{V}\right\|_{\mathcal{K}_{1}^{1}(\Omega)} \leq C\left\|u-u_{I}\right\|_{\mathcal{K}_{1}^{1}(\Omega)}
$$

The constant $C$ in the expression is independent of the triangulation $\mathcal{T}$ and of the solution $u$. From our estimates on the interpolation error $\left\|u-u_{I}\right\|_{\mathcal{K}_{1}^{1}(\Omega)}$, we shall construct a class $\mathcal{C}(l, h, \kappa, m, \epsilon)$ of partitions $\mathcal{T}$ of $\Omega=(0,1) \times(0, l), l>0$, such that

$$
\left\|u-u_{V}\right\|_{H^{1}(\Omega)} \leq C \operatorname{dim}(V)^{-m / 2}\|f\|_{H^{m-1}(\Omega)}, \quad \forall f \in H^{m-1}(\Omega) .
$$

More details about the notation and proof will be given in Section 4 .

In Section 5 numerical results will be presented for the operator $\mathcal{L}_{1}:=-\partial_{x}^{2}-$ $\frac{1}{x^{2}} \partial_{y}^{2}$ on $\hat{\Omega}:=(0,1) \times(0,10)$ with a smooth $f$. We will compare the rates of convergence of the numerical solutions for different mesh sizes. The convergence history will verify our theoretical prediction and demonstrate the efficiency of our technique to approximate the solution.

\section{Weighted Sobolev Spaces $\mathcal{K}_{a}^{m}$}

As explained above, weighted Sobolev spaces are convenient for the problem since the solution $u$ may not belong to $H^{m+1}(\Omega)$ for large $m$, even if $f \in \mathcal{C}^{\infty}(\Omega)$. In this section, we shall introduce the weighted Sobolev spaces $\mathcal{K}_{a}^{m}(\Omega)$ and establish some properties of them, which are useful for the study of the boundary value problem (11). One can refer to [11, 23, 34, 35, 36, for more information and additional results on Sobolev spaces with weights. 


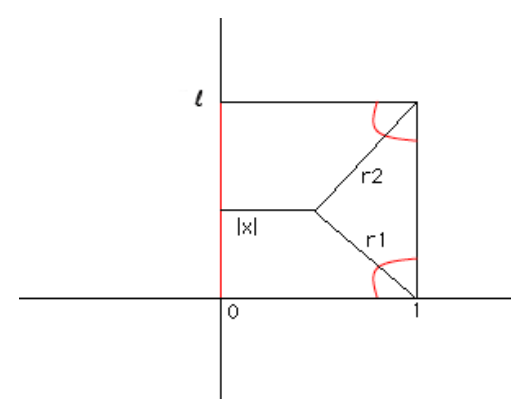

FIGURE 1. Domain $\Omega$ and distances.

2.1. Notation. As usual, the regular Sobolev spaces $H^{m}(\Omega)$ on the domain $\Omega$ for $m \in\{0,1,2, \ldots\}$ are defined as follows [26]:

$$
H^{m}(\Omega)=\left\{v \in L^{2}(\Omega), \partial^{\alpha} v \in L^{2}(\Omega), \forall|\alpha| \leq m\right\},
$$

with the multi-index $\alpha=\left(\alpha_{1}, \alpha_{2}\right) \in \mathbb{Z}_{+}^{2},|\alpha|:=\alpha_{1}+\alpha_{2}$ and $\partial^{\alpha}:=\partial^{\alpha_{1}} \partial^{\alpha_{2}}$. The $H^{m}$-norm of any $v \in H^{m}(\Omega)$ is defined by

$$
\|v\|_{H^{m}(\Omega)}^{2}:=\sum_{|\alpha| \leq m}\left\|\partial^{\alpha} v\right\|_{L^{2}(\Omega)}^{2},
$$

where the $L^{2}$-norm is

$$
\|v\|_{L^{2}(\Omega)}^{2}=\int_{\Omega}|v|^{2} d x d y .
$$

Let $X(x, y) \in \Omega$ be an arbitrary point in the domain $\Omega$. To define weighted Sobolev spaces $\mathcal{K}_{a}^{m}$ on the domain $\Omega$, we denote by $r_{1}(x, y)$ and $r_{2}(x, y)$ two smooth functions, such that $r_{1}(x, y)=$ the distance from $X(x, y)$ to $(1,0)$, if the distance $<\frac{1}{4} ; r_{2}(x, y)=$ the distance from $X(x, y)$ to $(1, l)$, if the distance $<\frac{1}{4} ; \frac{1}{4} \leq r_{1}, r_{2} \leq 1$ otherwise. In addition, we require that both $r_{1}(x, y)$ and $r_{2}(x, y)$ are equal to 1 if $x<\frac{1}{2}$. (Figure 1). The above distances are used in the definition of the spaces $\mathcal{K}_{a}^{m}$ and play an important role in reflecting the properties of the solution of equation (11) near the vertices $(1,0),(1, l)$ [14. Denote by $S=\{(1,0),(1, l),[0, y], 0 \leq y \leq l\}$ the set containing the vertices and the degenerate boundary of the domain. Then, we define the weighed Sobolev spaces $\mathcal{K}_{a}^{m}$ on $\Omega$ as follows.

Definition 2.1. Let $R:=\left\{x<\frac{1}{2}\right\} \cup\left\{r_{i}<\frac{1}{4}, i=1,2\right\} \subset \Omega$ be a subset of the domain. Let $\rho(x, y)$ be a positive smooth function, such that $\rho$ stands for the distance from $X(x, y)$ to the set $S$ for any $X(x, y) \in R$, and $\rho$ satisfies that $\frac{1}{4} \leq \rho \leq 1$ for any point in the region $\Omega \backslash R$. Therefore, $\rho=x$ in the neighborhood of the degenerate boundary; $\rho=r_{1}$ and $\rho=r_{2}$ in the neighborhoods of $(1,0)$ and $(1, l)$, respectively. Then, for $i, j, m \in\{0,1,2, \ldots\}$, the $m$ th weighted Sobolev space is

$$
\mathcal{K}_{a}^{m}(\Omega):=\left\{v \in L_{l o c}^{2}(\Omega), \rho^{-a}\left(r_{1} r_{2}\right)^{i+j}\left(x \partial_{x}\right)^{i} \partial_{y}^{j} v \in L^{2}(\Omega), i+j \leq m\right\} .
$$

The $\mathcal{K}_{a}^{m}$-norm for any function $v \in \mathcal{K}_{a}^{m}(\Omega)$ is

$$
\|v\|_{\mathcal{K}_{a}^{m}(\Omega)}^{2}:=\sum_{i+j \leq m}\left\|\rho^{-a}\left(r_{1} r_{2}\right)^{i+j}\left(x \partial_{x}\right)^{i} \partial_{y}^{j} v\right\|_{L^{2}(\Omega)}^{2} .
$$


By $L_{l o c}^{2}(\Omega)$, we mean the space that includes all the functions on $\Omega$, whose restriction to any compact subset $A \subset \Omega$ is in $L^{2}(A)$. Also, we denote by $H_{c}^{m}(\Omega)$ the space of functions that belong to $H^{m}(A)$ for any compact subset $A \subset \Omega$. In addition, we set $\Omega_{\xi}:=(0, \xi) \times(0, l) \subset \Omega, 0<\xi<1$, and $S_{\xi}:=\Omega \backslash \Omega_{\xi}$ to be two particular subsets that will be used very often in the text.

Since $\rho^{-a}\left(r_{1} r_{2}\right)^{i+j}\left(x \partial_{x}\right)^{i} \partial_{y}^{j} v \in L^{2}(\Omega), \forall v \in \mathcal{K}_{a}^{m}(\Omega)$, the completeness of the space $\mathcal{K}_{a}^{m}(\Omega)$ then follows the completeness of $L^{2}(\Omega)$ and integration by parts with standard arguments in [1, 26. Moreover, $\mathcal{K}_{a}^{m}(\Omega)$ is a Hilbert space associated with the inner product

$$
(u, v)_{\mathcal{K}_{a}^{m}}=\sum_{i+j \leq m} \int_{\Omega}\left(\rho^{-2 a}\left(r_{1} r_{2}\right)^{2(i+j)}\left(x \partial_{x}\right)^{i} \partial_{y}^{j} u\left(x \partial_{x}\right)^{i} \partial_{y}^{j} v\right) d x d y
$$

Recall that for regular Sobolev spaces $H^{m}(\Omega)$ [19, 26, 46, $m>0$, the space $H^{-m}(\Omega)$ is the dual space of $H_{0}^{m}(\Omega)$, where $H_{0}^{m}(\Omega)$ is defined [1] as follows,

$$
H_{0}^{m}(\Omega)=\left\{v \in H^{m}(\Omega),\left.\partial^{i} v\right|_{\partial \Omega}=0, i<m\right\} .
$$

Similarly, we denote by $\mathcal{K}_{-a}^{-m}(\Omega) \cong\left(\mathcal{K}_{a}^{m}(\Omega) \cap\left\{\left.v\right|_{\partial \Omega}=0\right\}\right)^{\prime}$ the dual space of $\mathcal{K}_{a}^{m}(\Omega) \cap$ $\left\{\left.v\right|_{\partial \Omega}=0\right\}$ with respect to the pivot space $L^{2}(\Omega)$,

$$
\|w\|_{\mathcal{K}_{-a}^{-m}(\Omega)}:=\sup _{v \in \mathcal{K}_{a}^{m}(\Omega) \cap\left\{\left.v\right|_{\partial \Omega}=0\right\}} \frac{\left|\int_{\Omega} v w\right|}{\|v\|_{\mathcal{K}_{a}^{m}(\Omega)}}, \quad v \neq 0 .
$$

We also agree that if $\|v\|_{\mathcal{K}_{a}^{m}(\Omega)}=\infty$, then $v$ is not in $\mathcal{K}_{a}^{m}(\Omega)$.

Remark 2.2. The parameters in the spaces $\mathcal{K}_{a}^{m}(\Omega)$ are related to the distances to the vertices $(1,0),(1, l)$ and to the degenerate boundary $x=0$, where one may see singularities of the solution. It is clear that equation (11) is strongly elliptic in the small neighborhoods of $(1,0)$ and $(1, l)$ with the coefficient frozen in $x$ at those points. In fact, near the vertices $(1,0)$ and $(1, l)$, the spaces $\mathcal{K}_{a}^{m}(\Omega)$ are the usual weighted Sobolev spaces for elliptic equations on corner singularities [9, 15, 34; while in the neighborhood of $x=0, \mathcal{K}_{a}^{m}(\Omega)$ can be considered as the usual weighted spaces in polar coordinates, by setting $r=x$ and $\theta=y$. Our weighted Sobolev spaces are invented in a way that is based on the property of the operator $\mathcal{L}_{\delta}$ and the geometry of the domain. For this reason, some properties of $\mathcal{K}_{a}^{m}(\Omega)$ are important for the study of the regularity of the solution and for the construction of the finite dimensional subspaces in the FEM.

Throughout this paper, our notation will follow what we have introduced in this subsection. Based on the definition of the weighted Sobolev space, we shall give some observations and lemmas for $\mathcal{K}_{a}^{m}(\Omega)$.

2.2. Lemmas. Here we summarize several properties for the spaces $\mathcal{K}_{a}^{m}(\Omega)$ that are useful for the development of the theorems in Section 3 and Section 4 . Most of the properties are derived from straightforward calculation based on the definition of $\mathcal{K}_{a}^{m}(\Omega)$. For the reason that most functions we are interested in are defined on $\Omega$, we shall omit $\Omega$ in the notation $\mathcal{K}_{a}^{m}(\Omega)$ and $H^{m}(\Omega)$, which are used often below, to simplify the expression. Therefore, $\mathcal{K}_{a}^{m}=\mathcal{K}_{a}^{m}(\Omega)$ and $H^{m}=H^{m}(\Omega)$ for the rest of this paper. Moreover, $a \simeq b$ means that there exist constants $C_{1}, C_{2}>0$, such that $C_{1} b \leq a \leq C_{2} b$. By an isomorphism, we shall mean a continuous bijection between two Banach spaces. As usual, we denote by $r$ and $\theta$ the corresponding variables in the polar coordinates. 
The first lemma claims an alternative definition for the space $\mathcal{K}_{a}^{m}$.

Lemma 2.3. Denote by $P_{x} \subset \Omega, P_{r_{1}} \subset \Omega$ and $P_{r_{2}} \subset \Omega$ the small neighborhoods of $x=0$ and the vertices $(1,0),(1, l)$, respectively. Then, for $P_{r}=P_{r_{1}} \cup P_{r_{2}}$, we have $\mathcal{K}_{a}^{m}=\left\{u \in H_{c}^{m}(\Omega), \rho^{-a}\left(r \partial_{r}\right)^{i} \partial_{\theta}^{j} u \in L^{2}\left(P_{r}\right), \rho^{-a}\left(x \partial_{x}\right)^{i} \partial_{y}^{j} u \in L^{2}\left(P_{x}\right), \forall i+j \leq m\right\}$.

Proof. On the outside of $P=P_{x} \cup P_{r}, u \in H^{m}(\Omega \backslash P)$ is equivalent to $u \in \mathcal{K}_{a}^{m}(\Omega \backslash P)$, since $\rho, r_{1}, r_{2}$ and $x$ are all bounded from above and bounded away from 0 by the definition.

On the region $P_{x}$, we notice $\rho=x$ and $r_{1}=r_{2}=1$. Therefore,

$$
\left\|\rho^{-a}\left(r_{1} r_{2}\right)^{i+j}\left(x \partial_{x}\right)^{i} \partial_{y}^{j} u\right\|_{L^{2}\left(P_{x}\right)}=\left\|\rho^{-a}\left(x \partial_{x}\right)^{i} \partial_{y}^{j} u\right\|_{L^{2}\left(P_{x}\right)} .
$$

On $P_{r_{i}}, i=1,2$, we freeze the coefficient of $\mathcal{L}_{\delta}$ in $x$ at the vertex and change the variables $x, y$ into $r, \theta$ for the polar coordinates centered at the vertex, then

$$
\begin{aligned}
& \partial_{x}=\cos (\theta) \partial_{r}-\frac{\sin (\theta)}{r} \partial_{\theta}, \\
& \partial_{y}=\sin (\theta) \partial_{r}+\frac{\cos (\theta)}{r} \partial_{\theta},
\end{aligned}
$$

where $\rho=r_{1}=r$ on $P_{r_{1}}$ and $\rho=r_{2}=r$ on $P_{r_{2}}$. The proof then follows from

$$
\begin{aligned}
& \left\|\rho^{-a} r^{i+j}\left(\cos (\theta) \partial_{r}-\frac{\sin (\theta)}{r} \partial_{\theta}\right)^{i}\left(\sin (\theta) \partial_{r}+\frac{\cos (\theta)}{r} \partial_{\theta}\right)^{j} u\right\|_{L^{2}\left(P_{r_{i}}\right)} \\
& \simeq\left\|\rho^{-a} \sum_{h+k \leq i+j}\left(r \partial_{r}\right)^{h} \partial_{\theta}^{k} u\right\|_{L^{2}\left(P_{r_{i}}\right)} .
\end{aligned}
$$

Lemma 2.4. The function $\rho^{-b}\left(r_{1} r_{2}\right)^{i+j}\left(x \partial_{x}\right)^{i} \partial_{y}^{j} \rho^{b}$ is bounded on $\Omega$.

Proof. On the region $P_{r}$ where $\rho=r_{1}=r$ or $\rho=r_{2}=r$, we follow the notation in Lemma 2.3, and change to the polar coordinates centered at the vertices. Since $\left(r \partial_{r}\right)^{k} r^{b}=b^{k} r^{b}$ and $\partial_{\theta} r=0$, we have

$$
\begin{aligned}
& \left|\rho^{-b}\left(r_{1} r_{2}\right)^{i+j}\left(x \partial_{x}\right)^{i} \partial_{y}^{j} \rho^{b}\right|=\left|r^{-b}\left(r_{1} r_{2}\right)^{i+j}\left(x \partial_{x}\right)^{i} \partial_{y}^{j} r^{b}\right| \\
& \leq C_{1}\left|r^{-b} r^{i+j} \partial_{x}^{i} \partial_{y}^{j} r^{b}\right| \\
& =C_{1}\left|r^{-b} r^{i+j}\left(\cos (\theta) \partial_{r}-\frac{\sin (\theta)}{r} \partial_{\theta}\right)^{i}\left(\sin (\theta) \partial_{r}+\frac{\cos (\theta)}{r} \partial_{\theta}\right)^{j} r^{b}\right| \\
& \leq C\left|r^{-b} \sum_{k+h \leq i+j}\left(r \partial_{r}\right)^{k} \partial_{\theta}^{h} r^{b}\right| \\
& \leq C\left|r^{-b} \sum_{k \leq i+j}\left(r \partial_{r}\right)^{k} r^{b}\right|=C\left|\sum_{k \leq i+j} b^{k}\right| .
\end{aligned}
$$

Therefore, $\rho^{-b}\left(r_{1} r_{2}\right)^{i+j}\left(x \partial_{x}\right)^{i} \partial_{y}^{j} \rho^{b}$ is bounded on $P_{r}$.

On the region $P_{x}$ where $\rho=x$, we have $\left(x \partial_{x}\right)^{i} \rho^{b}=b^{i} \rho^{b}$ and $\partial_{y} \rho=0$. Thus, the proof follows

$$
\begin{aligned}
\left|\rho^{-b}\left(r_{1} r_{2}\right)^{i+j}\left(x \partial_{x}\right)^{i} \partial_{y}^{j} \rho^{b}\right| & =\left|\rho^{-b}\left(x \partial_{x}\right)^{i} \partial_{y}^{j} \rho^{b}\right| \\
& =\left|\rho^{-b}\left(x \partial_{x}\right)^{i} \rho^{b}\right|=C\left|b^{i}\right| .
\end{aligned}
$$

As for $\Omega \backslash P$, the complement of $P=P_{x} \cup P_{r}$, since $\rho$ is smooth and bounded away from 0 , the function $\rho^{-b}\left(r_{1} r_{2}\right)^{i+j}\left(x \partial_{x}\right)^{i} \partial_{y}^{j} \rho^{b}$ is bounded. Thus, the proof is completed. 
Consequently, Lemma 2.4 shows

Lemma 2.5. For the spaces $\mathcal{K}_{a}^{m}, \rho^{b} \mathcal{K}_{a}^{m}=\mathcal{K}_{a+b}^{m}$, where $\rho^{b} \mathcal{K}_{a}^{m}=\left\{\rho^{b} v, \forall v \in \mathcal{K}_{a}^{m}\right\}$. Therefore, the multiplication by $\rho^{b}$ defines an isomorphism $\mathcal{K}_{a}^{m} \rightarrow \mathcal{K}_{a+b}^{m}$.

Proof. Let $v \in \mathcal{K}_{a}^{m}$ and $w=\rho^{b} v$. Then $\left|\rho^{-a}\left(r_{1} r_{2}\right)^{i+j}\left(x \partial_{x}\right)^{i} \partial_{y}^{j} v\right| \in L^{2}$, for $i+j \leq m$. Moreover, we have

$$
\begin{aligned}
\left|\rho^{-a-b}\left(r_{1} r_{2}\right)^{i+j}\left(x \partial_{x}\right)^{i} \partial_{y}^{j} w\right| & =\left|\rho^{-a-b}\left(r_{1} r_{2}\right)^{i+j}\left(x \partial_{x}\right)^{i} \partial_{y}^{j} \rho^{b} v\right| \\
& =\rho^{-a-b}\left(r_{1} r_{2}\right)^{i+j}\left|\sum_{k \leq i, h \leq j}\left(x \partial_{x}\right)^{k} \partial_{y}^{h} \rho^{b}\left(x \partial_{x}\right)^{i-k} \partial_{y}^{j-h} v\right| \\
& \leq C \sum_{k \leq i, h \leq j}\left|\rho^{-a}\left(r_{1} r_{2}\right)^{i+j-k-h}\left(x \partial_{x}\right)^{i-k} \partial_{y}^{j-h} v\right| \in L^{2} .
\end{aligned}
$$

The last inequality is the consequence of Lemma 2.4. Thus, $\rho^{b} \mathcal{K}_{a}^{m}$ is continuously embedded in $\mathcal{K}_{a+b}^{m}$. On the other hand, because this embedding holds for any real number $b$, we have the opposite

$$
\mathcal{K}_{a+b}^{m}=\rho^{b} \rho^{-b} \mathcal{K}_{a+b}^{m} \subset \rho^{b} \mathcal{K}_{a}^{m},
$$

which completes the proof.

Recall that $\Omega_{\xi}=(0, \xi) \times(0, l), 0<\xi<1$. From a direct verification based on the definitions of $H^{m}$ and $\mathcal{K}_{a}^{m}$, we can also derive the following lemma.

Lemma 2.6. We have $\mathcal{K}_{0}^{0}=L^{2}$ and for $m^{\prime} \leq m$, $a^{\prime} \leq a$,

1. $\mathcal{K}_{a}^{m} \subset \mathcal{K}_{a^{\prime}}^{m^{\prime}}$,

2. $\|u\|_{\mathcal{K}_{a^{\prime}}^{m^{\prime}}\left(\Omega_{\xi}\right)} \leq \xi^{a-a^{\prime}}\|u\|_{\mathcal{K}_{a}^{m}\left(\Omega_{\xi}\right)}, \forall u \in \mathcal{K}_{a}^{m}, \xi<\frac{1}{2}$.

Proof. The first item in the lemma is the result of the inequality below. For any $u \in \mathcal{K}_{a}^{m}$ and $m^{\prime} \leq m, a^{\prime} \leq a$,

$$
\sum_{i+j \leq m^{\prime}}\left\|\rho^{-a^{\prime}}\left(r_{1} r_{2}\right)^{i+j}\left(x \partial_{x}\right)^{i} \partial_{y}^{j} u\right\|_{L^{2}}^{2} \leq C \sum_{i+j \leq m}\left\|\rho^{-a}\left(r_{1} r_{2}\right)^{i+j}\left(x \partial_{x}\right)^{i} \partial_{y}^{j} u\right\|_{L^{2}}^{2} .
$$

Note that on $\Omega_{\xi}, \xi<\frac{1}{2}$, we have $\rho=x, r_{1}=r_{2}=1$. Then the second item in this lemma follows

$$
\begin{aligned}
\|u\|_{\mathcal{K}_{a^{\prime}}^{m^{\prime}}\left(\Omega_{\xi}\right)}^{2} & =\sum_{i+j \leq m^{\prime}}\left\|\rho^{-a^{\prime}}\left(r_{1} r_{2}\right)^{i+j}\left(x \partial_{x}\right)^{i} \partial_{y}^{j} u\right\|_{L^{2}\left(\Omega_{\xi}\right)}^{2} \\
& \leq \sum_{i+j \leq m}\left\|\rho^{-a^{\prime}}\left(r_{1} r_{2}\right)^{i+j}\left(x \partial_{x}\right)^{i} \partial_{y}^{j} u\right\|_{L^{2}\left(\Omega_{\xi}\right)}^{2} \\
& =\xi^{2\left(a-a^{\prime}\right)} \sum_{i+j \leq m}\left\|\xi^{a^{\prime}-a} \rho^{-a^{\prime}}\left(r_{1} r_{2}\right)^{i+j}\left(x \partial_{x}\right)^{i} \partial_{y}^{j} u\right\|_{L^{2}\left(\Omega_{\xi}\right)}^{2} \\
& \leq \xi^{2\left(a-a^{\prime}\right)} \sum_{i+j \leq m}\left\|\rho^{-a}\left(r_{1} r_{2}\right)^{i+j}\left(x \partial_{x}\right)^{i} \partial_{y}^{j} u\right\|_{L^{2}\left(\Omega_{\xi}\right)}^{2} \\
& =\xi^{2\left(a-a^{\prime}\right)}\|u\|_{\mathcal{K}_{a}^{m}\left(\Omega_{\xi}\right)}^{2} .
\end{aligned}
$$

We note that the weights defined in $\mathcal{K}_{a}^{m}$ only depend on the distances to certain parts of the boundary. From Lemma 2.3. we obtain that the $H^{m}$ - and $\mathcal{K}_{a}^{m}$-norm are equivalent on any compact subset $A \subset \Omega$. 
Lemma 2.7. Let $\xi$ be a positive number, and let $G \subset \Omega$ be an open subset such that $r \geq \xi$ on $G$. Then $\|u\|_{H^{m}(G)} \leq M_{1}\|u\|_{\mathcal{K}_{a}^{m}(G)}$ and $\|u\|_{\mathcal{K}_{a}^{m}(G)} \leq M_{2}\|u\|_{H^{m}(G)}$, for any $u \in H^{m}(G)$, where the constants $M_{1}$ and $M_{2}$ only depend on $\xi$ and $m$.

Proof. As stated in Lemma 2.3, the parameters $\rho, r_{1}, r_{2}$ and $x$ in the expression of $\mathcal{K}_{a}^{m}$-norm are all bounded from 0 on $G$, since the distance from $G$ to the set $S$ has a lower bound $\xi$. Then, the proof follows the definitions of the spaces and the norms involved.

The following lemma states the relation between $H^{m}$ and $\mathcal{K}_{a}^{m}$ on $\Omega$.

Lemma 2.8. On $\Omega,\|u\|_{H^{m}} \leq M_{1}\|u\|_{\mathcal{K}_{m}^{m}}$, and $\|u\|_{\mathcal{K}_{a}^{m}} \leq M_{2}\|u\|_{H^{m}}$ for $a \leq 0$, where $M_{1}$ and $M_{2}$ depend on $m$ and $a$.

Proof. This lemma is basically a consequence of the definitions of those norms. Recall $P, P_{x}$ and $P_{r}$ from Lemma 2.3. Then, based on $i+j \leq m$ and $\rho=x$ on $P_{x}$,

$$
\begin{aligned}
\|u\|_{\mathcal{K}_{m}^{m}\left(P_{x}\right)}^{2} & =\sum_{i+j \leq m}\left\|\rho^{-m}\left(x \partial_{x}\right)^{i} \partial_{y}^{j} u\right\|_{L^{2}\left(P_{x}\right)}^{2} \\
& \geq C \sum_{i+j \leq m}\left\|\partial_{x}^{i} \partial_{y}^{j} u\right\|_{L^{2}\left(P_{x}\right)}^{2}=C\|u\|_{H^{m}\left(P_{x}\right)}^{2} .
\end{aligned}
$$

On the other hand, we have

$$
\begin{aligned}
\|u\|_{\mathcal{K}_{m}^{m}\left(P_{r}\right)}^{2} & \geq C \sum_{i+j \leq m}\left\|\rho^{-m} \rho^{i+j} \partial_{x}^{i} \partial_{y}^{j} u\right\|_{L^{2}\left(P_{r}\right)}^{2} \\
& \geq C \sum_{i+j \leq m}\left\|\partial_{x}^{i} \partial_{y}^{j} u\right\|_{L^{2}\left(P_{r}\right)}^{2}=C\|u\|_{H^{m}\left(P_{r}\right)}^{2},
\end{aligned}
$$

based on $i+j \leq m$ and $\rho=r_{1}$ or $r_{2}$ on $P_{r}$.

For the region $\Omega \backslash P$, Lemma 2.7 shows that $\|u\|_{H^{m}(\Omega \backslash P)} \leq M_{1}\|u\|_{\mathcal{K}_{m}^{m}(\Omega \backslash P)}$, which completes the proof for the first argument in the lemma.

The second inequality can be proved in a similar way by comparing different norms on $P$ and $\Omega \backslash P$, which will be shown in Lemma 2.10.

From Lemma 2.5 and Lemma 2.8 we have the following corollary.

Corollary 2.9. We have $\mathcal{K}_{m+a}^{m} \subset \rho^{a} H^{m} \subset \mathcal{K}_{a}^{m}$.

The proof is based on the isomorphism arising from the multiplication in Lemma 2.5 and the inequalities in Lemma 2.8 .

The following lemma will compare $\mathcal{K}_{a}^{m}$ and $H^{m}$ near the $y$-axis and the vertices.

Lemma 2.10. Let $\xi$ be a positive number and let $G^{\prime}$ be an open subset of $\Omega$, such that $\rho<\xi$ on $G^{\prime}$. Then $\|u\|_{H^{m}\left(G^{\prime}\right)} \leq C_{1} \xi^{a-m}\|u\|_{\mathcal{K}_{a}^{m}\left(G^{\prime}\right)}$ if $a \geq m$, and $\|u\|_{\mathcal{K}_{a}^{m}\left(G^{\prime}\right)} \leq C_{2} \xi^{-a}\|u\|_{H^{m}\left(G^{\prime}\right)}$ if $a \leq 0$, where $C_{1}$ and $C_{2}$ are generic constants depending on $m$.

Proof. For $a \geq m$, we first have

$$
\|u\|_{H^{m}\left(G^{\prime}\right)} \leq C_{1}\|u\|_{\mathcal{K}_{m}^{m}\left(G^{\prime}\right)}
$$


from Lemma 2.8. Then, on the subregion of $G^{\prime}$ that is close to $x=0$, Lemma 2.6 shows

$$
\|u\|_{\mathcal{K}_{m}^{m}} \leq \xi^{a-m}\|u\|_{\mathcal{K}_{a}^{m}}
$$

For the subregion that is near one of the vertices, we have

$$
\begin{aligned}
\|u\|_{\mathcal{K}_{a}^{m}}^{2} & \geq C \sum_{i+j \leq m}\left\|\rho^{-a} \rho^{i+j} \partial_{x}^{i} \partial_{y}^{j} u\right\|_{L^{2}}^{2} \\
& \geq \xi^{2(m-a)}\left\|\partial_{x}^{i} \partial_{y}^{j} u\right\|_{L^{2}}^{2}=\xi^{2(m-a)}\|u\|_{H^{m}}^{2} .
\end{aligned}
$$

The last inequality is based on $\rho<\xi$ and $i+j \leq m$ on this subregion. Therefore, $\|u\|_{H^{m}\left(G^{\prime}\right)} \leq C_{1} \xi^{a-m}\|u\|_{\mathcal{K}_{a}^{m}\left(G^{\prime}\right)}$ for $a \geq m$ by combining the estimates on different subregions of $G^{\prime}$.

For $a \leq 0$, similarly, on the subregion of $G^{\prime}$, which is close to $x=0$, because $\rho=x, a \leq 0$ and $\rho<\xi$, we have the following inequalities:

$$
\begin{aligned}
\|u\|_{\mathcal{K}_{a}^{m}}^{2} & =\sum_{i+j \leq m}\left\|\rho^{-a}\left(x \partial_{x}\right)^{i} \partial_{y}^{j} u\right\|_{L^{2}}^{2} \\
& \leq C \xi^{-2 a} \sum_{i+j \leq m}\left\|\partial_{x}^{i} \partial_{y}^{j} u\right\|_{L^{2}}^{2}=C \xi^{-2 a}\|u\|_{H^{m}}^{2} .
\end{aligned}
$$

On the subregion close to one of the vertices, the inequalities are

$$
\begin{aligned}
\|u\|_{\mathcal{K}_{a}^{m}}^{2} & \leq C \sum_{i+j \leq m}\left\|\rho^{-a} \rho^{i+j} \partial_{x}^{i} \partial_{y}^{j} u\right\|_{L^{2}}^{2} \\
& \leq C \xi^{-2 a} \sum_{i+j \leq m}\left\|\partial_{x}^{i} \partial_{y}^{j} u\right\|_{L^{2}}^{2}=C \xi^{-2 a}\|u\|_{H^{m}}^{2} .
\end{aligned}
$$

Therefore, for $a \leq 0,\|u\|_{\mathcal{K}_{a}^{m}\left(G^{\prime}\right)} \leq C \xi^{-a}\|u\|_{H^{m}\left(G^{\prime}\right)}$. This also provides the proof of the second inequality in Lemma 2.8

We have derived several lemmas to reveal the relations between weighted Sobolev spaces $\mathcal{K}_{a}^{m}$ and regular Sobolev spaces $H^{m}$. They are the preliminaries for our main results in the next section. Now, we shall give an important lemma for the homogeneity of the norms of weighted Sobolev spaces $\mathcal{K}_{a}^{m}\left(\Omega_{\xi}\right)$. This is one of the main reasons that we use weighted Sobolev spaces for the analysis.

We define the dilation of a function on $\Omega_{\xi}:=(0, \xi) \times(0, l), 0<\xi<1$, first. For $0<\lambda<1$, let $G \subset \Omega_{\xi}$ be an open subset. Also, let $v$ be a function on $G$. Then, we define the dilation function $v_{\lambda}(x, y):=v(\lambda x, y)$ for any point $(x, y) \in G^{\prime} \subset \Omega_{\xi / \lambda}$, such that $(\lambda x, y) \in G$. The relation of the norms of $v$ and its dilation $v_{\lambda}$ is given by the following lemma.

Lemma 2.11. Let $G \subset \Omega_{\xi} \backslash \Omega_{\lambda \xi}$ be an open subset and $u(x, y)$ a function on $G$, $0<\lambda<1, \xi / \lambda<\frac{1}{2}$. Then $\left\|u_{\lambda}\right\|_{\mathcal{K}_{a}^{m}\left(G^{\prime}\right)}^{2}=\lambda^{2 a-1}\|u\|_{\mathcal{K}_{a}^{m}(G)}^{2}$ for any $u \in \mathcal{K}_{a}^{m}(G)$. This relation also holds for $G \subset \Omega_{\lambda \xi}, \xi<\frac{1}{2}$. 
Proof. Note that from the dilation, $G^{\prime} \subset \Omega_{\frac{1}{2}}$, and hence $\rho=x, r_{1}=r_{2}=1$ on $G^{\prime}$. The proof then follows by the change of variables and a direct calculation. Let $w=\lambda x$,

$$
\begin{aligned}
\left\|u_{\lambda}(x, y)\right\|_{\mathcal{K}_{a}^{m}\left(G^{\prime}\right)}^{2} & =\sum_{i+j \leq m} \int_{G^{\prime}}\left(x^{-a}\left(r_{1} r_{2}\right)^{i+j}\left(x \partial_{x}\right)^{i} \partial_{y}^{j} u_{\lambda}\right)^{2} d x d y \\
& =\sum_{i+j \leq m} \int_{G}\left(\lambda^{a} w^{-a}\left(w \partial_{w}\right)^{i} \partial_{y}^{j} u(w, y)\right)^{2} \frac{1}{\lambda} d w d y \\
& =\sum_{i+j \leq m} \int_{G} \lambda^{2 a-1}\left(w^{-a}\left(w \partial_{w}\right)^{i} \partial_{y}^{j} u(w, y)\right)^{2} d w d y \\
& =\lambda^{2 a-1} \sum_{i+j \leq m} \int_{G}\left(w^{-a}\left(w \partial_{w}\right)^{i} \partial_{y}^{j} u(w, y)\right)^{2} d w d y \\
& =\lambda^{2 a-1} \sum_{i+j \leq m}\left\|w^{-a}\left(w \partial_{w}\right)^{i} \partial_{y}^{j} u\right\|_{L^{2}(G)}^{2} \\
& =\lambda^{2 a-1}\|u\|_{\mathcal{K}_{a}^{m}(G)}^{2} .
\end{aligned}
$$

We note that the proof above can be carried out without any restriction on $G \subset \Omega_{\lambda \xi}$, $\xi<\frac{1}{2}$ as well. Therefore, this relation for $u$ and $u_{\lambda}$ also holds on this region.

The significance of Lemma 2.11 is that it shows the possibility to estimate the weighted norms on another region under dilation.

Let us explain why we considered Lemma 2.11 only on $\Omega_{\xi}$. Recall $P_{x}$ and $P_{r}$ defined in Lemma 2.3, Let $P=P_{x} \cup P_{r}$. Then, the strong ellipticity of the operator $\mathcal{L}_{\delta}$ on $\Omega \backslash P$ admits a unique solution $u \in H^{m+1}(\Omega \backslash P) \cap\left\{\left.u\right|_{\partial \Omega}=0\right\}$ for $f \in H^{m-1}$. Therefore, $u$ has no singularity on $\Omega \backslash P$, and no further study is needed in general on this region. In fact, Lemma 2.11 is particular for the analysis of the solution near $x=0$, while one can refer to [3, 14, 31] for the solution around the vertices $(1,0),(1, l)$.

We have listed several lemmas on $\mathcal{K}_{a}^{m}$ for the degenerate problem and we shall conclude this section with the following result.

Lemma 2.12. The operator $\mathcal{L}_{\delta}$ defines a continuous map $\mathcal{L}_{\delta}: \mathcal{K}_{a+1}^{m+1}(\Omega) \rightarrow$ $\mathcal{K}_{a-1}^{m-1}(\Omega)$.

Proof. We need to show that for any $u \in \mathcal{K}_{a+1}^{m+1}(\Omega),\left\|\mathcal{L}_{\delta} u\right\|_{\mathcal{K}_{a-1}^{m-1}} \leq C\|u\|_{\mathcal{K}_{a+1}^{m+1}}$ on $P_{r}, P_{x}$ and $\Omega \backslash P$, which are defined in Lemma 2.3 .

On $\Omega \backslash P, \mathcal{L}_{\delta}=-\partial_{x}^{2}-\frac{\delta^{2}}{x^{2}} \partial_{y}^{2}$ is strongly elliptic. Therefore, it is a bounded operator $H^{m+1}(\Omega \backslash P) \rightarrow H^{m-1}(\Omega \backslash P)[26$. Then, the argument for this lemma follows the equivalence of the spaces $H^{m+1}(\Omega \backslash P)$ and $\mathcal{K}_{a}^{m+1}(\Omega \backslash P)$.

On $P_{r}$, let $g=r_{1} r_{2}, H^{m}=H^{m}\left(P_{r}\right)$ and $\mathcal{K}_{a}^{m}=\mathcal{K}_{a}^{m}\left(P_{r}\right)$ in the proof for simplicity. Then, based on $g \simeq \rho$, the following inequalities hold with the coefficient frozen in $x$ at 1 , 


$$
\begin{aligned}
& \left\|\mathcal{L}_{\delta} u\right\|_{\mathcal{K}_{a-1}^{m-1}} \leq C_{0} \sum_{i+j \leq m-1}\left\|\rho^{1-a} g^{i+j}\left(x \partial_{x}\right)^{i} \partial_{y}^{j}\left(-\partial_{x}^{2} u-\frac{\delta^{2}}{x^{2}} \partial_{y}^{2} u\right)\right\|_{L^{2}} \\
& =C_{0} \sum_{i+j \leq m-1}\left\|\rho^{1-a} g^{i+j} \partial_{x}^{i} \partial_{y}^{j}\left(-\partial_{x}^{2} u-\delta^{2} \partial_{y}^{2} u\right)\right\|_{L^{2}} \\
& \leq C_{1} \sum_{i+j \leq m-1}\left(\left\|\rho^{1-a} g^{i+j} \partial_{x}^{i+2} \partial_{y}^{j} u\right\|_{L^{2}}+\left\|\rho^{1-a} g^{i+j} \partial_{x}^{i} \partial_{y}^{j+2} u\right\|_{L^{2}}\right) \\
& \leq C_{2} \sum_{i+j \leq m-1}\left(\left\|\rho^{-a-1} g^{i+j+2} \partial_{x}^{i+2} \partial_{y}^{j} u\right\|_{L^{2}}+\left\|\rho^{-a-1} g^{i+j+2} \partial_{x}^{i} \partial_{y}^{j+2} u\right\|_{L^{2}}\right) \\
& \leq C\|u\|_{\mathcal{K}_{a+1}^{m+1}} .
\end{aligned}
$$

On $P_{x}$, similarly, let $H^{m}=H^{m}\left(P_{x}\right)$ and $\mathcal{K}_{a}^{m}=\mathcal{K}_{a}^{m}\left(P_{x}\right)$ in the proof for simplicity. Then, based on $\rho=x, r_{1}=r_{2}=1$, we have the estimates below:

$$
\begin{aligned}
& \left\|\mathcal{L}_{\delta} u\right\|_{\mathcal{K}_{a-1}^{m-1}} \leq C_{1} \sum_{i+j \leq m-1}\left\|\rho^{1-a}\left(x \partial_{x}\right)^{i} \partial_{y}^{j}\left(-\partial_{x}^{2} u-\frac{\delta^{2}}{x^{2}} \partial_{y}^{2} u\right)\right\|_{L^{2}} \\
& \leq C_{1} \sum_{i+j \leq m-1}\left(\left\|x^{1-a}\left(x \partial_{x}\right)^{i} \partial_{x}^{2} \partial_{y}^{j} u\right\|_{L^{2}}+\left\|x^{1-a}\left(x \partial_{x}\right)^{i} \partial_{y}^{j+2} \frac{\delta^{2}}{x^{2}} u\right\|_{L^{2}}\right) \\
& \leq \quad C_{2} \sum_{i+j \leq m-1}\left(\left\|x^{1-a}\left(\left(x \partial_{x}\right)^{i+2}-\left(x \partial_{x}\right)^{i+1}\right) x^{-2} \partial_{y}^{j} u\right\|_{L^{2}}\right. \\
& \left.\quad+\left\|x^{-a-1}\left(-2+x \partial_{x}\right)^{i} \partial_{y}^{j+2} u\right\|_{L^{2}}\right) \\
& \leq \quad C_{2} \sum_{i+j \leq m-1}\left(\left\|x^{-a-1}\left(-2+x \partial_{x}\right)^{i+2} \partial_{y}^{j} u\right\|_{L^{2}}\right. \\
& \left.\left.\quad+\| x^{-a-1}\left(-2+x \partial_{x}\right)^{i+1}\right) \partial_{y}^{j} u\left\|_{L^{2}}+\right\| x^{-a-1}\left(-2+x \partial_{x}\right)^{i} \partial_{y}^{j+2} u \|_{L^{2}}\right) \\
& \leq C\|u\|_{\mathcal{K}_{a+1}^{m+1}}
\end{aligned}
$$

We here use $x^{-a}\left(x \partial_{x}\right)^{i} x^{a} u=\left(a+x \partial_{x}\right)^{i} u$ to simplify the expression.

In the next section, we will show that this map $\mathcal{L}_{\delta}: \mathcal{K}_{a+1}^{m+1} \cap\left\{\left.u\right|_{\partial \Omega}=0\right\} \rightarrow \mathcal{K}_{a-1}^{m-1}$ is a bijection, if the index $a$ satisfies some conditions.

\section{The Well-POSEDNESS AND REGUlARITY OF THE SOLUTION}

First, we need the following estimates on the solution of equation (11). Recall that we defined the set $S=\{(1,0),(1, l),[0, y], 0 \leq y \leq l\}$ in Section 2 It has been shown in 2, 3, 14, that the trace or restriction of $u \in \mathcal{K}_{a}^{m}$ on the boundary follows

$$
\left.u\right|_{\partial \Omega \backslash S} \in \mathcal{K}_{a-\frac{1}{2}}^{m-\frac{1}{2}}(\partial \Omega \backslash S) .
$$

However, the estimate of the trace on $x=0$ is needed to derive the corresponding bilinear form $a(\cdot, \cdot)$ for the boundary value problem (11), although the trace on this part of boundary is generally undefined in the weighted Sobolev spaces $\mathcal{K}_{a}^{m}$ [9, 14, 34. We note that for $m \geq 1, \mathcal{K}_{m}^{m} \subset H^{m}$ by Lemma 2.8. Denote each 
segment of $\partial \Omega$ by $\bar{D}_{i}, i=1,2,3,4$, where $D_{i}$ is open. In particular, let $D_{1}:=(0, y)$, $0 \leq y \leq l$, be the corresponding open set for the degenerate boundary $x=0$. Then, the trace

$$
\left.u\right|_{D_{i}} \in H^{m-\frac{1}{2}}\left(D_{i}\right), \quad \forall u \in \mathcal{K}_{m}^{m}(\Omega),
$$

is defined [31. Consequently, for $u \in \mathcal{K}_{1}^{1}$, the trace of $u$ is well defined in $L^{2}$ on every $D_{i}$ of the boundary $\partial \Omega$. Furthermore, we can show that $\left.u\right|_{D_{1}}=0$ for $u \in \mathcal{K}_{1}^{1}$.

Lemma 3.1. For any function $u \in \mathcal{K}_{1}^{1}$, its trace on $D_{i}$ is well defined in $L^{2}$ and, moreover, we have $\left.u\right|_{D_{1}}=0$. consequently, the corresponding bilinear form for equation (11) is $a(u, v)=\int_{\Omega}\left(\partial_{x} u \partial_{x} v+\frac{\delta^{2}}{x^{2}} \partial_{y} u \partial_{y} v\right) d x d y, \forall v \in \mathcal{K}_{1}^{1}(\Omega) \cap\left\{\left.v\right|_{\partial \Omega}=0\right\}$.

Proof. For $u \in \mathcal{K}_{1}^{1}$, the trace $\left.u\right|_{D_{i}}$ belongs to $H^{\frac{1}{2}}\left(D_{i}\right)$ by the arguments above, hence in $L^{2}\left(D_{i}\right)$.

Moreover, on $\Omega_{\xi}=(0, \xi) \times(0, l), \xi<1 / 2$, since $\rho=x$, we have

$$
\frac{1}{\xi^{2}} \int_{\Omega_{\xi}} u^{2} d x d y \leq \int_{\Omega_{\xi}} \frac{1}{x^{2}} u^{2} d x d y \leq C\|u\|_{\mathcal{K}_{1}^{1}\left(\Omega_{\xi}\right)}^{2}<\infty .
$$

Therefore, $\int_{\Omega_{\xi}} u^{2} d x d y \rightarrow 0$ as $\xi \rightarrow 0$. Hence, the trace $\left.u\right|_{D_{1}}=0$ in $L^{2}$ by continuity.

Thus, the following bilinear form is obtained by integration by parts,

$$
a(u, v)=\int_{\Omega}\left(\partial_{x} u \partial_{x} v+\frac{\delta^{2}}{x^{2}} \partial_{y} u \partial_{y} v\right) d x d y, \quad \forall v \in \mathcal{K}_{1}^{1}(\Omega) \cap\left\{\left.v\right|_{\partial \Omega}=0\right\} .
$$

Now, we shall prove the existence and uniqueness of the solution of equation (1) in weighted Sobolev spaces.

Theorem 3.2. On $\Omega$, the map $\mathcal{L}_{\delta}: \mathcal{K}_{1}^{m+1}(\Omega) \cap\left\{\left.u\right|_{\partial \Omega}=0\right\} \rightarrow \mathcal{K}_{-1}^{m-1}(\Omega)$ is an isomorphism, for $\delta>0, m \geq 0$. Namely, there is a unique solution $u \in \mathcal{K}_{1}^{m+1}(\Omega) \cap$ $\left\{\left.u\right|_{\partial \Omega}=0\right\}$ for equation (1) if $f \in \mathcal{K}_{-1}^{m-1}(\Omega)$.

Proof. We shall first prove it for $m=0$. From Lemma 3.1 we have the following weak formulation for equation (11),

$a(u, v)=\int_{\Omega}\left(\partial_{x} u \partial_{x} v+\frac{\delta^{2}}{x^{2}} \partial_{y} u \partial_{y} v\right) d x d y=\int_{\Omega} f v d x d y, \quad \forall v \in \mathcal{K}_{1}^{1}(\Omega) \cap\left\{\left.v\right|_{\partial \Omega}=0\right\}$.

Then, we shall show the equivalence between the energy norm induced by $a(\cdot, \cdot)$ and the $\mathcal{K}_{1}^{1}$-norm $\|\cdot\|_{\mathcal{K}_{1}^{1}(\Omega)}$ to complete the proof.

Based on the definitions of $a(\cdot, \cdot)$ and the $\mathcal{K}_{1}^{1}$-norm on $\Omega$, the continuity of $a(\cdot, \cdot)$ can be verified as follows. From the Hölder inequality, there exists a constant $C$, not depending on $u$ or $v$, such that $a(u, v) \leq C \mid\|u\|_{\mathcal{K}_{1}^{1}}\|v\|_{\mathcal{K}_{1}^{1}}$. Therefore, $a(\cdot, \cdot)$ is a continuous (bounded) bilinear form on $\mathcal{K}_{1}^{1}$.

To prove the coercivity, we adopt the following notation. Let $\Omega_{\xi}=(0, \xi) \times(0, l)$ be the rectangular domain near the boundary $x=0$. Denote by $B(v, r)$ the open ball of radius $r$ centered at $v$. For any of the vertices $v_{1}=(1,0), v_{2}=(1, l)$, let $\Omega_{r_{\xi_{i}}}=\Omega \cap B\left(v_{i}, \xi\right), i=1,2$, be the corresponding conical domain, such that $\Omega_{r_{\xi_{i}}}$ can be characterized in polar coordinates by

$$
\Omega_{r_{\xi_{i}}}=\left\{(r, \theta) \mid 0<r<\xi, 0<\theta<\frac{\pi}{2}\right\} .
$$


Note that $a(u, u)$ is equivalent to $|u|_{\mathcal{K}_{1}^{1}}^{2}=\sum_{i+j=1}\left\|\rho^{-1}\left(r_{1} r_{2}\right)\left(x \partial_{x}\right)^{i} \partial_{y}^{j} u\right\|_{L^{2}}^{2}$ by their definitions. Then, we shall first prove the following weaker inequalities for $\xi$ small:

$$
\begin{gathered}
\int_{\Omega_{\xi}} \frac{u^{2}}{x^{2}} d x d y \leq C \int_{\Omega_{\xi}}\left(\partial_{x} u\right)^{2}+\frac{\delta^{2}}{x^{2}}\left(\partial_{y} u\right)^{2} d x d y, \\
\int_{\Omega_{r_{\xi_{i}}}} \frac{u^{2}}{r^{2}} d x d y \leq C \int_{\Omega_{r_{\xi_{i}}}}\left(\partial_{x} u\right)^{2}+\delta^{2}\left(\partial_{y} u\right)^{2} d x d y,
\end{gathered}
$$

since $\rho=x$ and $\rho=r$ on $\Omega_{\xi}$ and $\Omega_{r_{\xi_{i}}}$, respectively.

On the domain $\Omega_{\xi}$, we first have the one-dimensional Poincaré inequality for $y$,

$$
\int_{0}^{l} u^{2} d y \leq C_{1} \int_{0}^{l}\left(\partial_{y} u\right)^{2} d y .
$$

By integrating with respect to $x$, we obtain

$$
\int_{\Omega_{\xi}} \frac{u^{2}}{x^{2}} d y d x \leq C_{1} \int_{\Omega_{\xi}} \frac{\left(\partial_{y} u\right)^{2}}{x^{2}} d y d x \leq C \int_{\Omega_{\xi}}\left(\partial_{x} u\right)^{2}+\frac{\delta^{2}}{x^{2}}\left(\partial_{y} u\right)^{2} d y d x,
$$

where $C$ is independent of $u$.

Similarly, we have the one-dimensional Poincaré inequality for $\theta$ on $\Omega_{r_{\xi_{i}}}$,

$$
\int_{0}^{\frac{\pi}{2}} u^{2} d \theta \leq C_{1} \int_{0}^{\frac{\pi}{2}}\left(\partial_{\theta} u\right)^{2} d \theta
$$

By integrating in polar coordinates, we have

$$
\int_{\Omega_{r_{\xi_{i}}}} \frac{u^{2}}{r^{2}} d x d y=\int_{\Omega_{r_{\xi_{i}}}} \frac{u^{2}}{r} d r d \theta \leq C_{1} \int_{\Omega_{r_{\xi_{i}}}} \frac{\left(\partial_{\theta} u\right)^{2}}{r} d r d \theta .
$$

Since $\int_{\Omega_{r_{\xi_{i}}}}\left(\partial_{x} u\right)^{2}+\left(\partial_{y} u\right)^{2} d x d y=\int_{\Omega_{r_{\xi_{i}}}} r\left(\partial_{r} u\right)^{2}+\frac{\left(\partial_{\theta} u\right)^{2}}{r} d r d \theta$, we now have

$$
\int_{\Omega_{r_{\xi_{i}}}} \frac{u^{2}}{r^{2}} d x d y \leq C \int_{\Omega_{r_{\xi_{i}}}}\left(\partial_{x} u\right)^{2}+\delta^{2}\left(\partial_{y} u\right)^{2} d x d y
$$

with $C$ independent of $u$.

Let $\Omega_{r_{\xi}}:=\Omega_{r_{\xi_{1}}} \cup \Omega_{r_{\xi_{2}}}$. Thus, based on the usual Poincaré inequality in $\Omega \backslash\left(\Omega_{\xi} \cup\right.$ $\Omega_{r_{\xi}}$ ) and the inequalities above, we complete the proof for the coercivity $a(u, u) \geq$ $C\|u\|_{\mathcal{K}_{1}^{1}(\Omega)}^{2}$. Then, the existence of the unique solution $u \in \mathcal{K}_{1}^{1}(\Omega) \cap\left\{\left.u\right|_{\partial \Omega}=0\right\}$ follows the Lax-Milgram Theorem [19].

For $m \geq 1$, the proof follows [14, 33], which is based on the regularity of the solution derived by the Mellin transform on an infinite domain.

As an extension from this theorem, one has the following corollary.

Corollary 3.3. There exists a constant $\eta>0$, depending on $\Omega$, such that

$$
\mathcal{L}_{\delta}: \mathcal{K}_{1+\epsilon}^{m+1}(\Omega) \cap\left\{\left.u\right|_{\partial \Omega}=0\right\} \rightarrow \mathcal{K}_{-1+\epsilon}^{m-1}(\Omega)
$$

is an isomorphism for $0<|\epsilon|<\eta$.

Proof. Denote by $\mathcal{L}_{\delta \epsilon}$ the operator defined by $\mathcal{L}_{\delta}$ but on the space $\mathcal{K}_{1+\epsilon}^{m+1} \cap\left\{\left.u\right|_{\partial \Omega}=\right.$ $0\}$. Then, from Theorem 3.2 and Lemma 2.5 (see the diagram below), the operator $\mathcal{L}_{\delta \epsilon}$ is an isomorphism if, and only if

$$
\mathcal{A}_{\delta \epsilon}:=\rho^{-\epsilon} \mathcal{L}_{\delta \epsilon} \rho^{\epsilon}: \mathcal{K}_{1}^{m+1} \cap\left\{\left.u\right|_{\partial \Omega}=0\right\} \rightarrow \mathcal{K}_{-1}^{m-1}
$$


is an isomorphism.

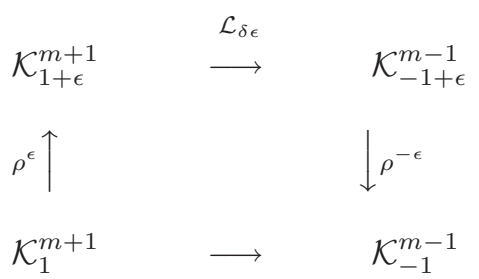

Then the proof follows the fact that $\mathcal{A}_{\delta \epsilon}$ is a continuous bijection as $\epsilon=0$ and the operator $\mathcal{A}_{\delta \epsilon}$ depends continuously in norm on the parameter $\epsilon$.

Remark 3.4. For a brief summary, we have taken the advantage of weighed spaces $\mathcal{K}_{a}^{m}$ to prove the well-posedness of the solution $u \in \mathcal{K}_{1}^{m+1} \cap\left\{\left.u\right|_{\partial \Omega}=0\right\}$ of equation (11), for all $f \in \mathcal{K}_{-1}^{m-1}$. Furthermore, there exists some constant $\eta$, such that $\mathcal{L}_{\delta}$ is still invertible on weighted spaces that depend on $\eta$. In fact, one will find that it is important to know the exact upper bound of $\epsilon$ in the FEM. To be more precise, $\eta$ is determined by the local behavior of the solution for equation (11) near the set $S=\{(1,0),(1, l),[0, y], 0 \leq y \leq l\}$. Recall $\Omega_{\xi}=(0, \xi) \times(0, l)$ and $\Omega_{r_{\xi}}=\Omega_{r_{\xi_{1}}} \cup \Omega_{r_{\xi_{2}}}$ in Theorem 3.2. Then, it is possible to show that $\eta_{1}=\frac{\sqrt{l^{2}+4 \delta^{2} \pi^{2}}}{2 l}$ on $\Omega_{\xi}$ for $\xi<1 / 2$. Namely, $\mathcal{A}_{\delta \epsilon}: \mathcal{K}_{1}^{m+1}\left(\Omega_{\xi}\right) \cap\left\{\left.u\right|_{y=0, l}=0\right\} \rightarrow \mathcal{K}_{-1}^{m-1}\left(\Omega_{\xi}\right)$ is invertible for $|\epsilon|<\eta_{1}=\frac{\sqrt{l^{2}+4 \delta^{2} \pi^{2}}}{2 l}$. Here, we include some arguments on operator $\mathcal{A}_{\delta \epsilon}$ and the constant $\eta$.

We focus on the region $\Omega_{\xi}=(0, \xi) \times(0, l)$ for $\xi<1 / 2$ first. The indicial family of $\mathcal{A}_{\delta \epsilon}=\rho^{-\epsilon} \mathcal{L}_{\delta \epsilon} \rho^{\epsilon}$ for $\mathcal{K}_{1}^{m+1}\left(\Omega_{\xi}\right) \cap\left\{\left.u\right|_{y=0, l}=0\right\}$ is

$$
\left(i \tau+\epsilon+\frac{1}{2}\right)\left(i \tau+\epsilon-\frac{1}{2}\right)+\delta^{2} \partial_{y}^{2}
$$

acting on $H^{2}([0, l]) \cap\left\{\left.u\right|_{y=0, l}=0\right\}$. The eigenvalues of $\partial_{y}^{2}$ on $H^{2}([0, l]) \cap\left\{\left.u\right|_{y=0, l}=\right.$ $0\}$ are $-\left(\frac{k \pi}{l}\right)^{2}$ for $k \in \mathbb{N}:=\{1,2,3, \ldots\}$. On $\Omega_{r_{\xi_{i}}}$, the indicial family of $\mathcal{A}_{\delta \epsilon}$ for the vertex can be derived in a similar way as in [15, 40, by the Mellin transform 33 . Then, we have the corresponding eigenvalues that can be calculated numerically. Based on Kondratiev's results [34, the operater $\mathcal{A}_{\delta \epsilon}: \mathcal{K}_{1}^{m+1} \cap\left\{\left.u\right|_{\partial \Omega}=0\right\} \rightarrow \mathcal{K}_{-1}^{m-1}$ is Fredholm if, and only if its indicial family for the degenerate boundary $x=0$ and the indicial families for the two vertices are invertible for all $\tau \in \mathbb{R}$. This is seen to be the case for $\left(i \tau+\epsilon+\frac{1}{2}\right)\left(i \tau+\epsilon-\frac{1}{2}\right)+\delta^{2} \partial_{y}^{2}$, unless $\epsilon= \pm \frac{\sqrt{l^{2}+4 k^{2} \delta^{2} \pi^{2}}}{2 l}, k \in \mathbb{N}$.

Let $\eta_{1}=\frac{\sqrt{l^{2}+4 \delta^{2} \pi^{2}}}{2 l}$. Denote by $\eta_{2}$ the smallest positive value, such that one of the indicial families of $\mathcal{A}_{\delta \epsilon}$ for the vertices is not invertible as $\epsilon=\eta_{2}$. Then, $\mathcal{A}_{\delta \epsilon}=\rho^{-\epsilon} \mathcal{L}_{\delta \epsilon} \rho^{\epsilon}: \mathcal{K}_{1}^{m+1} \cap\left\{\left.u\right|_{\partial \Omega}=0\right\} \rightarrow \mathcal{K}_{-1}^{m-1}$ is Fredholm of index 0 when $|\epsilon|<\eta=\min \left(\eta_{1}, \eta_{2}\right)$, since $\mathcal{A}_{\delta \epsilon}$ is Fredholm of index 0 as $\epsilon=0$. Moreover, we note that the kernels of the operators $\mathcal{A}_{\delta \epsilon}$ are decreasing as $\epsilon$ is increasing, we conclude that they are invertible for $0 \leq \epsilon<\eta$. By taking the adjoint, we obtain the invertibility of $\mathcal{A}_{\delta \epsilon}$ for $-\eta<\epsilon \leq 0$ as well.

As the conclusion of the arguments above, we can take $\eta=\min \left(\eta_{1}, \eta_{2}\right)$, such that for any $|\epsilon|<\eta$, the operator $\mathcal{L}_{\delta}: \mathcal{K}_{1+\epsilon}^{m+1} \cap\left\{\left.u\right|_{\partial \Omega}=0\right\} \rightarrow \mathcal{K}_{-1+\epsilon}^{m-1}$ is still an isomorphism.

For values of $\epsilon$ outside the range above, the operator $\mathcal{A}_{\delta \epsilon}$ will no longer be invertible. In fact, it will have a non-zero index that can be computed using the 
results of [38. To compute $\eta_{2}$ for $\Omega_{r_{\xi}}$, one can follow the method in [6, 15, 31, and similar examples will be presented in a forthcoming paper.

\section{The Finite ELEMENT METHoD}

In this section, we consider the numerical approximation for the solution $u$ of equation (1D) by using the FEM. We shall provide a simple and explicit construction of a sequence of finite dimensional subspaces $V_{n} \subset H_{0}^{1}$ for equation (1), with possible singular solutions, such that the finite element solution $u_{n} \in V_{n}$ satisfies (Theorem 4.7)

$$
\left\|u-u_{n}\right\|_{H^{1}(\Omega)} \leq C \operatorname{dim}\left(V_{n}\right)^{-m / 2}\|f\|_{H^{m-1}(\Omega)}, \quad \forall f \in H^{m-1}(\Omega),
$$

which recovers the asymptotic rate of convergence for elliptic equations with highregulary solutions [19].

4.1. Estimate for the interpolation. Let us recall the following notation and the well-known approximation result (2) in order to carry out further analysis.

Suppose the bilinear form $a(\cdot, \cdot)$ for an equation is both continuous and coercive on $H^{1}$ for a star-shaped two-dimensional domain $D$. Let $V=V(\mathcal{T}, m+1)$ be the finite element space associated to the degree $m$ Lagrange triangle. Assume that all triangles $T_{j}$ of the triangulation $\mathcal{T}$ of domain $D$ have angles $\geq \alpha$ and edges of length $\leq h$ and $\geq a h$ (quasi-uniform triangles). Let $u_{V} \in V$ be the finite element solution determined by the weak form, and $u_{I} \in V$ the nodal interpolant of the continuous solution $u$, namely, $u_{I}\left(x_{i}\right)=u\left(x_{i}\right)$ on any node.

Then, there exist positive constants $c$ and $C_{1}=C_{1}(\alpha, m)$ such that

$$
c\left\|u-u_{V}\right\|_{H^{1}(D)} \leq\left\|u-u_{I}\right\|_{H^{1}(D)} \leq C_{1} h^{m}\|u\|_{H^{m+1}(D)}
$$

for all $u \in H^{m+1}(D), m \geq 1$. (See [19, 21.) The constant $c$ depends only on the bilinear form $a(\cdot, \cdot)$ by Céa's Lemma, while $C_{1}$ is independent of the solution. Note $u_{I}$ is well defined for $u \in H^{m+1}(D), m \geq 1$, by the Sobolev embedding theorem.

Let $M:=C_{1}(\alpha, m) M_{1} M_{2}$, where $M_{1}$ and $M_{2}$ are from Lemma 2.7. Then, we have the following estimate for the error $\left\|u-u_{I}\right\|_{\mathcal{K}_{1}^{1}}$ on a subset of $\Omega$. Note that on any compact subset $D \subset \Omega, u_{I}$ is also well defined for any $u \in \mathcal{K}_{a}^{m+1}, m \geq 1$, since the $H^{m+1}$ space and the $\mathcal{K}_{a}^{m+1}$ space are equivalent by Lemma 2.3 and Lemma 2.7

Theorem 4.1. Fix $\alpha>0$ and $0<\xi<1 / 4$. Let $P \subset \Omega$ be a polygonal domain, such that $\rho>\xi$ on $P$. Let $\mathcal{T}=\left\{T_{j}\right\}$ be a triangulation of $P$ with angles $\geq \alpha$ and sides $\leq h$. Then

$$
\left\|u-u_{I}\right\|_{\mathcal{K}_{1}^{1}(P)} \leq M h^{m}\|u\|_{\mathcal{K}_{1+\epsilon}^{m+1}(P)},
$$

for all $u \in \mathcal{K}_{1+\epsilon}^{m+1}(P)$, where $M$ depends on $\xi$ and $\alpha$.

Proof. The proof for the error estimate follows (2) and the equivalence of the $H^{m}$ and the $\mathcal{K}_{a}^{m}$-norms on $P$ immediately.

We point out here that the estimate in Theorem 4.1 does not extend to the entire domain $\Omega$ in general. It is reasonable to expect singularities for $u$ as it is approaching the degenerate boundary $x=0$, since it only belongs to the weighted Sobolev spaces instead of the usual Sobolev spaces. Also, it is possible that we have corner singularities near the vertices away from $x=0$, depending on the parameter $\delta$ and on the interior angle of the corner. In either of the cases, the constant $M$ cannot be uniformly bounded, which will destroy the optimal rate of convergence 
44 5. The FEM for elliptic equations with Dirichlet boundary condition near the corners has been widely studied [6, 11, 15, 39] in different principles. The basic idea is to reflect the singularities in finite elements by using anisotropic meshes near the corners. In those graded meshes, the size and shape of the triangles are carefully designed without increasing the number of degrees of freedom. Then, the optimal rate of convergence can be recovered in terms of the dimension of the space $V_{n}$. Therefore, from now on, we shall concentrate on the estimate near the degenerate boundary $x=0$, since we have been quite clear for the corners.

For a triangle $T$ in the mesh, we let $h_{x}^{\prime}$ be the length of the perpendicular projection of $T$ on the $x$-axis, and $h_{y}^{\prime}$ the length of the perpendicular projection on the $y$-axis. Recall $\Omega_{\xi}=(0, \xi) \times(0, l)$. Then, the extension of Theorem 4.1 on the thin rectangular region $\Omega_{\xi} \backslash \Omega_{\kappa \xi}$ is as follows.

Theorem 4.2. Let $\Omega_{\xi}=(0, \xi) \times(0, l)$ be a subset of $\Omega$ with $\xi<1 / 4$. Given $0<\kappa<1$, let $\mathcal{T}=\left\{T_{j}\right\}$ be a triangulation for the rectangular region $U:=\Omega_{\xi} \backslash \Omega_{\kappa \xi}$. Define $h_{x}:=\max _{T_{j}}\left\{h_{x}^{\prime}\right\}$ and $h_{y}:=\max _{T_{j}}\left\{h_{y}^{\prime}\right\}$ for all triangles in $\mathcal{T}$. Recall $V=V(\mathcal{T}, m+1)$ and the nodal interpolant $u_{I} \in V$ of the solution. Then,

$$
\left\|u-u_{I}\right\|_{\mathcal{K}_{1}^{1}(U)} \leq C(\kappa) \xi^{\epsilon}\left(h_{y}+\frac{h_{x}}{2 \xi}\right)^{m}|| u \|_{\mathcal{K}_{1+\epsilon}^{m+1}(U)},
$$

for all $u \in \mathcal{K}_{1+\epsilon}^{m+1}, m \geq 1, \epsilon>0$.

Proof. We first note that by the definitions of $h_{x}$ and $h_{y}$, in $U$, the mesh size (size of triangles $) \leq h_{y}+h_{x}$. Recall the dilation function $u_{\lambda}(x, y)=u(\lambda x, y)$ from Section 2 and note that $u_{I \lambda}=u_{\lambda I}$. Namely, dilation commutes with interpolation. Then, if we let $\lambda=2 \xi$ and $U^{\prime}=\left(\frac{\kappa}{2}, 1 / 2\right) \times(0, l)$, by Lemma 2.11 and Theorem 4.1, we have

$$
\begin{aligned}
\left\|u-u_{I}\right\|_{\mathcal{K}_{1}^{1}(U)} & =\lambda^{-\frac{1}{2}}\left\|u_{\lambda}-u_{I \lambda}\right\|_{\mathcal{K}_{1}^{1}\left(U^{\prime}\right)} \\
& =\lambda^{-\frac{1}{2}}\left\|u_{\lambda}-u_{\lambda I}\right\|_{\mathcal{K}_{1}^{1}\left(U^{\prime}\right)} \\
& \leq \lambda^{-\frac{1}{2}} M\left(h_{y}+\frac{h_{x}}{\lambda}\right)^{m}\left\|u_{\lambda}\right\|_{\mathcal{K}_{1}^{m+1}\left(U^{\prime}\right)} \\
& =M\left(h_{y}+\frac{h_{x}}{\lambda}\right)^{m}\|u\|_{\mathcal{K}_{1}^{m+1}(U)} \\
& \leq M \xi^{\epsilon}\left(h_{y}+\frac{h_{x}}{2 \xi}\right)^{m}\|u\|_{\mathcal{K}_{1+\epsilon}^{m+1}(U)} .
\end{aligned}
$$

The last inequality is based on Lemma 2.6 in Section 2 .

This theorem provides us the interpolation error for $u \in \mathcal{K}_{1+\epsilon}^{m+1}$ on a rectangular strip $U$ near $x=0$. Based on our observation above, it is possible to construct a class $\mathcal{C}(l, h, \kappa, m, \epsilon)$ of partitions $\mathcal{T}$ of $\Omega$, such that the optimal convergence rate is obtained.

Before we define the class $\mathcal{C}(l, h, \kappa, m, \epsilon)$, we assume that, near the vertices $(1,0)$ and $(1, l)$, a proper graded mesh has already been chosen to recover the optimal convergence rate, which is reasonable, based on our previous discussions. For this reason, we will not consider the graded mesh near the vertices in the definition below. In fact, we will use a uniform mesh near the corners to demonstrate our method to generate triangles. However, it is only for the purpose of simplifying the expressions. One needs to keep in mind that the uniform mesh near the corners in 
the following definition will be replaced by an appropriate graded mesh generally in practical computation. See [6, 11, 15, 39] and references therein.

4.2. Construction of the mesh. We shall introduce the construction of a class $\mathcal{C}(l, h, \kappa, m, \epsilon)$ of triangulations and the finite element spaces $V_{n}$ associated to it. In the class $\mathcal{C}(l, h, \kappa, m, \epsilon)$, we try to evenly distribute the interpolation error, and keep the same number of triangles as in the usual mid-point triangulation.

In the notation $\mathcal{C}(l, h, \kappa, m, \epsilon)$, we denote by $h$ the size of triangles in the triangulation on $\left(\frac{1}{2}, \frac{3}{4}\right) \times(0, l) \subset \Omega ; \kappa$ is the parameter to control the decay of triangles near $x=0$. We focus only on $\Omega_{\xi}=(0, \xi) \times(0, l)$, for $\xi<\frac{1}{2}$. (Graded meshes for both of the vertices $(1,0),(1, l)$ are needed in general, but it will have the same number of triangles as in the uniform mesh.) Here we define the class $\mathcal{C}(l, h, \kappa, m, \epsilon)$ for our problem.

Definition 4.3. For a fixed $m \in\{1,2, \ldots\}, \epsilon \in(0,1], l>0, h>0$, we define $\mathcal{C}(l, h, \kappa, m, \epsilon)$ to be the following set of triangulations. First, for a positive integer $N$ (the number of refinements), choose $\kappa$, such that

$$
\kappa^{N \epsilon} \leq C(l) h^{m} .
$$

Then, starting with three initial triangles in $\Omega \backslash \Omega_{\frac{1}{2}}$ (Figure 2), we decompose $\Omega_{\frac{1}{2}}$ into rectangular subdomains $\Omega^{0}:=\Omega_{\frac{1}{2}} \backslash \Omega_{\frac{\kappa}{2}}, \Omega^{1}:=\Omega_{\frac{\kappa}{2}} \backslash \Omega_{\frac{\kappa^{2}}{2}}, \ldots, \Omega^{j}:=\Omega_{\frac{\kappa^{j}}{2}} \backslash \Omega_{\frac{\kappa^{j}+1}{2}}$, for $j=0,1,2, \ldots, N-1$ and $\Omega^{N}:=\Omega_{\frac{\kappa^{N}}{2}}=\left(0, \frac{\kappa^{N}}{2}\right) \times(0, l)$. Note $h=l$ in the initial mesh. As in Theorem 4.2, we denote by $h_{x_{j}}$ and $h_{y_{j}}$ the maximum lengths of perpendicular projections of triangles in $\Omega^{j}$ on the $x$ - and $y$-axis, respectively. In the $j$ th refinement, $1 \leq j<N$, we triangulate $\Omega^{j-1}$ with three triangles (Figure 2), such that they satisfy

$$
h_{x_{j-1}}=\kappa^{j-1} / 2-\kappa^{j} / 2, \text { and } h_{y_{j-1}}=l .
$$

Meanwhile, new triangles are generated in $\Omega \backslash \Omega_{\frac{\kappa j-1}{2}}$, by connecting the mid-points of the old triangles. Note that there is no triangle in $\Omega^{i}, i \geq j$, yet. We simply repeat this process for $\Omega^{j}$ and $\Omega \backslash \Omega_{\frac{\kappa}{2}}$ in the next step. In the $N$ th refinement, besides generating triangles for $\Omega^{N-1}$ and $\Omega \backslash \Omega_{\frac{\kappa^{N-1}}{2}}$, we divide $\Omega^{N}$ into two triangles by the diagonal of the rectangle. Then, after $N$ refinements, the triangulation $\mathcal{T}$ is the union of the triangles in the triangulations of all the subdomains.

We now state the following property for the $\operatorname{class} \mathcal{C}(l, h, \kappa, m, \epsilon)$ we defined above.

Theorem 4.4. For each $m \geq 1$, there exists a constant $C$, such that

$$
\left\|u-u_{I}\right\|_{\mathcal{K}_{1}^{1}(\Omega)} \leq C h^{m}\|u\|_{\mathcal{K}_{1+\epsilon}^{m+1}(\Omega)}
$$

for any triangulation $\mathcal{T}$ in $\mathcal{C}(l, h, \kappa, m, \epsilon)$ and $u \in \mathcal{K}_{1+\epsilon}^{m+1}(\Omega) \cap\left\{\left.u\right|_{\partial \Omega}=0\right\}, \epsilon>0$.

The proof of this theorem needs the estimate on every $\Omega^{j}, j<N$ and $\Omega^{N}$, since it holds for $\Omega \backslash \Omega_{\frac{1}{2}}$ by our assumption. Due to the construction of the class $\mathcal{C}(l, h, \kappa, m, \epsilon)$, we present the following lemma for the last region $\Omega^{N}=\left(0, \frac{\kappa^{N}}{2}\right) \times$ $(0, l)$ first.

Lemma 4.5. On $\Omega^{N}=\left(0, \frac{\kappa^{N}}{2}\right) \times(0, l)$, from the construction of $\mathcal{C}(l, h, \kappa, m, \epsilon)$, the estimate on the error gives

$$
\left\|u-u_{I}\right\|_{\mathcal{K}_{1}^{1}\left(\Omega^{N}\right)} \leq C h^{m}\|u\|_{\mathcal{K}_{1+\epsilon}^{m+1}\left(\Omega^{N}\right)},
$$



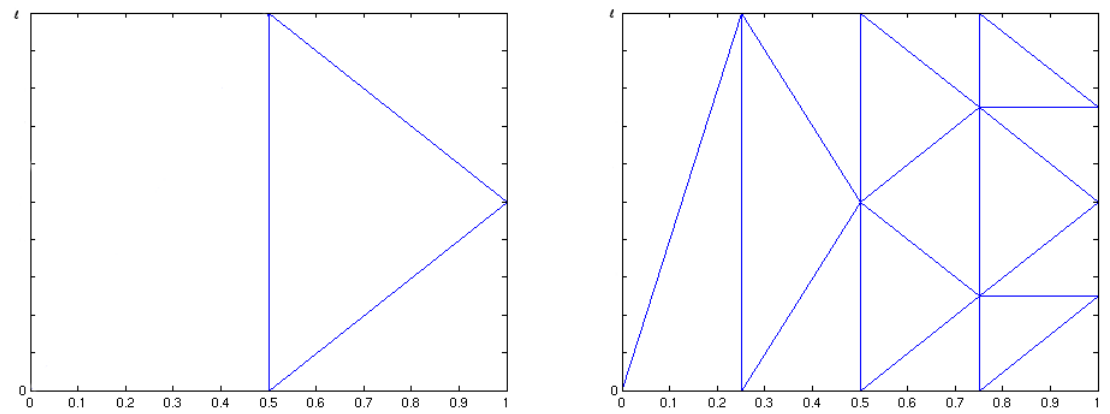

Figure 2. The initial mesh with three triangles (left); the triangulation after one refinement.

for all $u \in \mathcal{K}_{1+\epsilon}^{m+1}(\Omega) \cap\left\{\left.u\right|_{\partial \Omega}=0\right\}, \epsilon>0, m \geq 1$, where $C$ depends on $m$ and $\kappa$.

Proof. The proof follows the dilation of $u$ and the introduction of an auxiliary function $v$. We define the dilation $u_{\lambda}(x, y)=u(\lambda x, y)$ for $(\lambda x, y) \in \Omega^{N}$. Let $\lambda=\kappa^{N}$. Then, $u_{\lambda}(x, y) \in \mathcal{K}_{1+\epsilon}^{1+m}\left(\Omega_{\frac{1}{2}}\right)$ by Lemma 2.11. Meanwhile, let $\chi: \Omega_{\frac{1}{2}} \rightarrow[0,1]$ be a non-decreasing smooth function of $x$, which is equal to 0 in a neighborhood of $x=0$, but is equal to 1 at all the nodal points that do not lie on $x=0$. Then we introduce the auxiliary function $v=\chi u_{\lambda}$ on $\Omega_{\frac{1}{2}}$. Consequently, for a fixed $m$ and the corresponding nodal points in the triangulation, we have

$$
\begin{aligned}
\|v\|_{\mathcal{K}_{1}^{m+1}\left(\Omega_{\frac{1}{2}}\right)}^{2} & =\left\|\chi u_{\lambda}\right\|_{\mathcal{K}_{1}^{m+1}\left(\Omega_{\frac{1}{2}}\right)}^{2} \\
& =\sum_{i+j \leq m+1}\left\|\sum_{k \leq i} x^{-1}\left(r_{1} r_{2}\right)^{i+j}\left(x \partial_{x}\right)^{i-k} \partial_{y}^{j} u_{\lambda}\left(x \partial_{x}\right)^{k} \chi\right\|_{L^{2}\left(\Omega_{\frac{1}{2}}\right)}^{2} \\
& \leq C\left\|u_{\lambda}\right\|_{\mathcal{K}_{1}^{m+1}\left(\Omega_{\frac{1}{2}}\right)}^{2},
\end{aligned}
$$

where $C$ depends on $m$ and the function $\chi$. Moreover, one notes that the nodal interpolation $v_{I}=u_{\lambda I}$ on $\Omega_{\frac{1}{2}}$ by the definition of $v$.

Therefore, the proof is completed by the following inequalities:

$$
\begin{aligned}
\left\|u-u_{I}\right\|_{\mathcal{K}_{1}^{1}\left(\Omega^{N}\right)} & =\lambda^{-1 / 2}\left\|u_{\lambda}-v+v-u_{\lambda I}\right\|_{\mathcal{K}_{1}^{1}\left(\Omega_{\frac{1}{2}}\right)} \\
& \leq \lambda^{-1 / 2}\left(\left\|u_{\lambda}-v\right\|_{\mathcal{K}_{1}^{1}\left(\Omega_{\frac{1}{2}}\right)}+\left\|v-u_{\lambda I}\right\|_{\mathcal{K}_{1}^{1}\left(\Omega_{\frac{1}{2}}\right)}\right) \\
& \leq \lambda^{-1 / 2}\left(C_{1}\left\|u_{\lambda}\right\|_{\mathcal{K}_{1}^{1}\left(\Omega_{\frac{1}{2}}\right)}+C_{2} h_{y_{N}}^{m}\|v\|_{\mathcal{K}_{1}^{m+1}\left(\Omega_{\frac{1}{2}}\right)}\right) \\
& \leq \lambda^{-1 / 2}\left(C_{1}\left\|u_{\lambda}\right\|_{\mathcal{K}_{1}^{1}\left(\Omega_{\frac{1}{2}}\right)}+C_{3} h_{y_{N}}^{m}\left\|u_{\lambda}\right\|_{\mathcal{K}_{1}^{m+1}\left(\Omega_{\frac{1}{2}}\right)}\right) \\
& =C_{1}\|u\|_{\mathcal{K}_{1}^{1}\left(\Omega^{N}\right)}+C_{3} h_{y_{N}}^{m}\|u\|_{\mathcal{K}_{1}^{m+1}\left(\Omega^{N}\right)} \\
& \leq C_{4}\left(\frac{\kappa^{N}}{2}\right)^{\epsilon}\|u\|_{k_{1+\epsilon}^{m}\left(\Omega^{N}\right)}+C_{5} h_{y_{N}}^{m}\left(\frac{\kappa^{N}}{2}\right)^{\epsilon}\|u\|_{\mathcal{K}_{1+\epsilon}^{m+1}\left(\Omega^{N}\right)} \\
& \leq C h^{m}\|u\|_{\mathcal{K}_{1+\epsilon}^{m+1}\left(\Omega^{N}\right)} .
\end{aligned}
$$


The first and the fifth are from Lemma 2.11 the third and the fourth are the results of Theorem 4.1 and the relation between $v$ and $u_{\lambda}$; the sixth and the seventh are based on the construction of the triangulation.

Here we provide the proof for Theorem 4.4 by summing up the estimates on every region $\Omega^{j}$ for $j \leq N$.

Proof. Since we assume the estimate is valid on $\Omega \backslash \Omega_{\frac{1}{2}}$ that contains the vertices, it is sufficient to show that the estimate still holds on $\Omega_{\frac{1}{2}}$ for completing the proof.

The basic idea is to establish the estimate $\left\|u-u_{I}\right\|_{\mathcal{K}_{1}^{1}}$ on every $\Omega^{j}$ for $j=$ $0,1,2, \ldots, N-1$ and on $\Omega^{N}$.

On every $\Omega^{j}$, based on the construction of the triangulation, $\xi=\frac{\kappa^{j}}{2}$ for Theorem 4.2. Then, we have

$$
\frac{h_{x_{j}}}{2 \dot{\xi}} \simeq h_{y_{j}}=\frac{l}{2^{N-(j+1)}}
$$

Recall that $h$ represents the size of triangles in the region $\left(\frac{1}{2}, \frac{3}{4}\right) \times(0, l)$. Then $h=O\left(l / 2^{N}\right)$ after $N$ successive refinements. Since $\kappa^{N \epsilon} \leq C h^{m}$, by Theorem 4.2. we have for every $m$,

$$
\begin{aligned}
\left\|u-u_{I}\right\|_{\mathcal{K}_{1}^{1}\left(\Omega^{j}\right)} & \leq C \kappa^{j \epsilon}\left(\frac{l}{2^{N-(j+1)}}\right)^{m}\|u\|_{\mathcal{K}_{1+\epsilon}^{m+1}\left(\Omega^{j}\right)} \\
& \leq C h^{m}\|u\|_{\mathcal{K}_{1+\epsilon}^{m+1}\left(\Omega^{j}\right)} .
\end{aligned}
$$

As for the last region $\Omega^{N}$, we have $\left\|u-u_{I}\right\|_{\mathcal{K}_{1}^{1}\left(\Omega^{N}\right)} \leq C h^{m}\|u\|_{\mathcal{K}_{1+c}^{m+1}\left(\Omega^{N}\right)}$ by Lemma 4.5. The proof of Theorem 4.4 then follows by adding the squares of all these norms on $\Omega^{j}$ and $\Omega^{N}$.

Recall $\eta$ in Remark 3.4. From this point, we assume that $0<\epsilon<\min (1, \eta)$ is chosen such that

$$
\mathcal{L}_{\delta}: \mathcal{K}_{1+\epsilon}^{m+1} \cap\left\{\left.u\right|_{\partial \Omega}=0\right\} \rightarrow \mathcal{K}_{-1+\epsilon}^{m-1}
$$

is an isomorphism, which is possible due to Corollary 3.3 and Remark 3.4. Denote by $V$ the finite element space associated to the degree $m$ Lagrange triangle on the triangulation in Definition 4.3. Let $u_{V} \in V$ be the finite element solution of equation (11) determined by

$$
a\left(u_{V}, v_{V}\right)=\left(f, v_{V}\right), \quad \forall v_{V} \in V,
$$

where $a(\cdot, \cdot)$ is defined in Lemma 3.1. Then, we have the following estimate on $\left\|u-u_{V}\right\|_{H^{1}(\Omega)}$.

Theorem 4.6. Let $u \in \mathcal{K}_{1+\epsilon}^{m+1} \cap\left\{\left.u\right|_{\partial \Omega}=0\right\}$ be the solution for equation (11), $0<\epsilon<\min (1, \eta)$. Then, for each $m \geq 1$, there exists a constant $C$, such that

$$
\left\|u-u_{V}\right\|_{H_{1}^{1}(\Omega)} \leq C h^{m}\|f\|_{H^{m-1}(\Omega)}
$$

for any $\mathcal{T} \in \mathcal{C}(l, h, \kappa, m, \epsilon)$ and $\forall f \in H^{m-1}$. 
Proof. We denote by $\gamma_{\delta}$ the norm of the inverse operator $\mathcal{L}_{\delta}^{-1}: \mathcal{K}_{-1+\epsilon}^{m-1} \rightarrow \mathcal{K}_{1+\epsilon}^{m+1} \cap$ $\left\{\left.u\right|_{\partial \Omega}=0\right\}$. The theorem can be proved by the following inequalities:

$$
\begin{aligned}
\left\|u-u_{V}\right\|_{H^{1}} & \leq M\left\|u-u_{V}\right\|_{\mathcal{K}_{1}^{1}} \\
& \leq C_{1} M\left\|u-u_{I}\right\|_{\mathcal{K}_{1}^{1}} \\
& \leq C_{2} M h^{m}\|u\|_{\mathcal{K}_{1+\epsilon}^{m+1}} \\
& \leq C_{2} M \gamma_{\delta} h^{m}\|f\|_{\mathcal{K}_{-1+\epsilon}^{m-1}} \\
& \leq C h^{m}\|f\|_{H^{m-1}} .
\end{aligned}
$$

The first and fifth inequalities are from Lemma 2.8 the second inequality is based on Céa's Lemma and the third inequality is from Theorem 4.4 the fourth inequality is obtained by the invertibility of the operator $\mathcal{L}_{\delta}: \mathcal{K}_{1+\epsilon}^{m+1} \cap\left\{\left.u\right|_{\partial \Omega}=0\right\} \rightarrow \mathcal{K}_{-1+\epsilon}^{m-1}$.

We have proved our theorems based on an explicit construction of the class $\mathcal{C}(l, h, \kappa, m, \epsilon)$ for $\Omega$. Our estimates on the error were expressed by $h$, the size of those triangles in $\left(\frac{1}{2}, \frac{3}{4}\right) \times(0, l)$, as in the usual quasi-uniform finite element spaces. However, since there is no uniform size for the triangles in the triangulation, it is better to formulate the estimate in terms of the dimension of the finite dimensional subspace $V$. Based on the structure of the mesh we developed above, we attain the rate of convergence for the finite element solution $u_{V} \in V$ as follows.

Theorem 4.7. Let $u \in \mathcal{K}_{1+\epsilon}^{m+1} \cap\left\{\left.u\right|_{\partial \Omega}=0\right\}$ be the solution for equation (11), $0<\epsilon<\min (1, \eta)$. There exists a constant $C=C(l, \kappa, h, m, \epsilon)$ for $m \geq 1$, such that

$$
\left\|u-u_{V}\right\|_{H_{1}^{1}(\Omega)} \leq C \operatorname{dim}(V)^{-m / 2}\|f\|_{H^{m-1}(\Omega)}
$$

for any partition $\mathcal{T} \in \mathcal{C}(l, h, \kappa, m, \epsilon)$ and $\forall f \in H^{m-1}(\Omega)$.

Proof. Let $Q_{j}$ and $Q_{j-1}$ be the numbers of the elements in the meshes after $j$ and $j-1$ refinements from the initial mesh, respectively. Then, based on our construction, we have $Q_{j}=4 \times Q_{j-1}+3$, hence, $Q_{N}=O\left(4^{N}\right)$. On the other hand, the size of the triangles in $\left(\frac{1}{2}, \frac{3}{4}\right) \times(0, l)$ satisfies $h=O\left(2^{-N}\right)$, after $N$ levels of refinement. Thus, $\operatorname{dim}(V) \approx Q_{N} \approx h^{-2}$ for every $m \geq 1$. From Theorem 4.6, we have the following estimate in terms of the dimension of $V$ :

$$
\left\|u-u_{V}\right\|_{H_{1}^{1}(\Omega)} \leq C \operatorname{dim}(V)^{-m / 2}\|f\|_{H^{m-1}(\Omega)} .
$$

This is also the optimal convergence rate of the finite element solution expected for a smooth solution.

Based on the estimates for the interpolation error in weighted Sobolev spaces, we have designed a class of triangulations, on which the optimal rate of convergence is attained. In the next section, our theoretical results will be verified by comparing the convergence rates numerically in triangulations with different parameters $\kappa$.

\section{Numerical Results}

Here we present the numerical results to demonstrate our method to approximate the solution. The following model problem in the case $\delta=1$ and the domain $\hat{\Omega}=(0,1) \times(0,10)$ for equation (1) is considered:

$$
\left\{\begin{aligned}
-\partial_{x}^{2} u-\frac{1}{x^{2}} \partial_{y}^{2} u & =1 & & \text { in } \hat{\Omega}, \\
u & =0 & & \text { on } \quad \partial \hat{\Omega} .
\end{aligned}\right.
$$



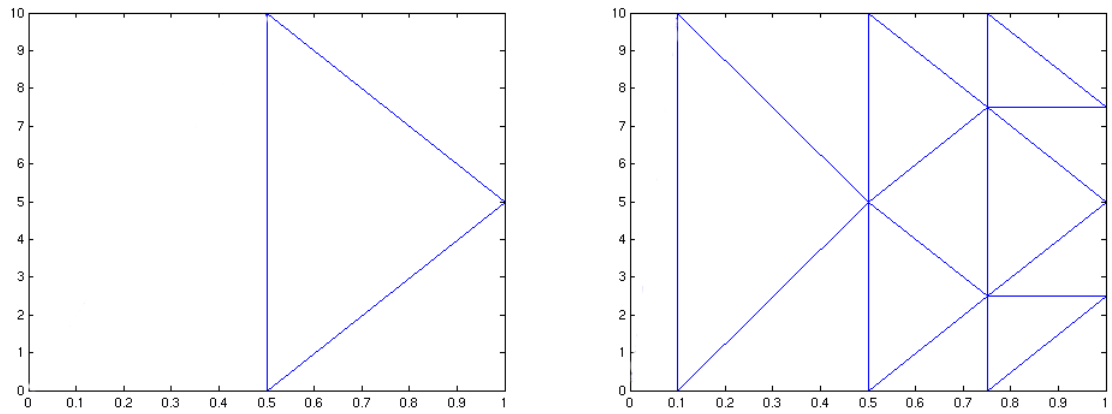

Figure 3. The initial mesh with three triangles (left); the mesh after one refinement $\kappa=0.2$ (right).

We also have chosen $m=1$, namely, piecewise linear functions for the FEM, because the implementation is then simpler, while the results are still relevant. From the previous theorems, the solution is not automatically in $H^{2}(\hat{\Omega})$ near the degenerate boundary. In fact, a more accurate a priori estimate in the usual Sobolev spaces $H^{m}(\hat{\Omega})$ shows that $u \in H^{s}(\hat{\Omega})$ for $s<1+\frac{\sqrt{100+4 \pi^{2}}}{20} \approx 1.59$ 34]. Note that the operator $\mathcal{L}_{1}$ is actually the Laplace operator $-\Delta$ near the vertices $(0,1),(0, l)$. The solution near those two corners, therefore, behaves like

$$
u(r, \theta)=r^{2 k} \zeta(\theta), \quad k \in \mathbb{Z}_{+},
$$

in polar coordinates [31, where the function $\zeta$ is smooth, only depending on $\theta$. For this reason, the solution has no singularity near those two vertices in $H^{2}$. The vertices $(1,0),(1, l)$ do not affect the regularity of the solution in this case. Consequently, it is not necessary to apply graded mesh there. Moreover, with a direct calculation based on our arguments in Section [3, we have $\eta_{1}=\frac{\sqrt{100+4 \pi^{2}}}{20}$ and $\eta_{2}=2$ on $\hat{\Omega}$. Then, one can set $0<\epsilon<\eta=\min \left(\eta_{1}, \eta_{2}\right)=\frac{\sqrt{100+4 \pi^{2}}}{20} \approx 0.59$ and take $0<\kappa=2^{-1 / \epsilon}$ for the graded mesh near the degenerate boundary $x=0$. Therefore, we have the range $\kappa<2^{-1 / 0.59} \approx 0.309$, on which the optimal rate of convergence in Theorem 4.7 holds for the model problem.

To construct the mesh on which we have the convergence rate as in Theorem 4.7. we start with three initial triangles (Figure 3). In every step of refinement, we pick two points $\left(x_{1}, 0\right)$ and $\left(x_{2}, 10\right)$ as two vertices of the new triangle and the third vertex of the new triangle is placed at the mid-piont of the base of the old triangle. Denote by $d$ the minimum distance from any point in the old triangles to $x=0$. Then, the parameter $\kappa$ controls the position of the new points, such that $x_{1}=x_{2}, \kappa=x_{1} / d$. Meanwhile, other new triangles are generated on the region that is enclosed by old triangles, by the mid-points as described in the previous section (Figure 3). Therefore, the new triangle that is approaching $x=0$ is specially designed to fulfill the requirement in Definition 4.4, while all the other new triangles are generated by connecting the mid-points of the old triangles. In the last step, the last region $\hat{\Omega}_{\frac{\kappa N}{2}}$ is divided into two triangles by the diagonal of the rectangle, as described in Section 4 


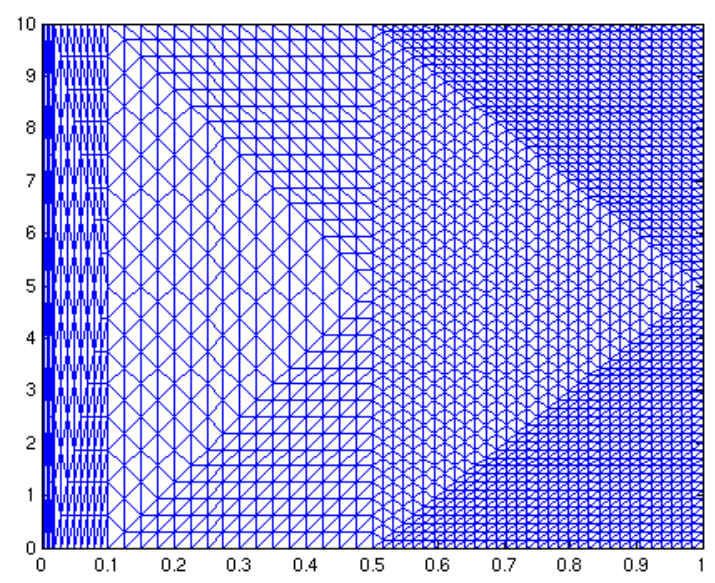

Figure 4. The mesh after 5 levels of refinements for $\kappa=0.2$.

One also notes that the triangles near the degenerate boundary are getting thinner and thinner in our construction, in which the maximum-angle condition is apparently violated. Nevertheless, the difficulty is already overcome by Theorem 4.2 and Theorem 4.4 .

The finest mesh in our numerical experiments is obtained after 10 successive refinements of the coarsest mesh and has roughly $2^{22} \approx 4 \times 10^{6}$ elements. The preconditioned conjugate gradient (PCG) method is used to solve the resulting system of algebraic equations.

We have tested several values of the parameter $\kappa$ for the model problem. The convergence rates are summarized in Table 1. These results convincingly show that the theoretical approximation order can be verified in practical calculations with $\kappa<0.3$.

TABLE 1. Convergence history with $e=\log _{2}\left(\frac{\left|u_{j}-u_{j-1}\right|_{\mathcal{K}_{1}^{1}}}{\left|u_{j+1}-u_{j}\right|_{\mathcal{K}_{1}^{1}}^{1}}\right)$.

\begin{tabular}{|c|lllllll|}
\hline$j$ & $e: \kappa=0.1$ & $e: \kappa=0.2$ & $e: \kappa=0.25$ & $e: \kappa=0.35$ & $e: \kappa=0.4$ & $e: \kappa=0.5$ \\
\hline 2 & 0.45 & 0.46 & 0.43 & 0.35 & 0.31 & 0.22 \\
\hline 3 & 0.66 & 0.63 & 0.59 & 0.48 & 0.42 & 0.31 \\
\hline 4 & 0.82 & 0.78 & 0.73 & 0.61 & 0.54 & 0.40 \\
\hline 5 & 0.92 & 0.88 & 0.84 & 0.71 & 0.63 & 0.47 \\
\hline 6 & 0.97 & 0.94 & 0.89 & 0.76 & 0.68 & 0.52 \\
\hline 7 & 0.99 & 0.96 & 0.93 & 0.79 & 0.71 & 0.55 \\
\hline 8 & 1.00 & 0.98 & 0.94 & 0.81 & 0.73 & 0.56 \\
\hline 9 & 1.00 & 0.99 & 0.96 & 0.82 & 0.74 & 0.57 \\
\hline
\end{tabular}

The left most column in Table 1 shows the number of the refinement level, and $u_{j}$ denotes the numerical solution corresponding to the mesh after $j$ levels of refinement. The quantity printed out in other columns in the table represents the 
convergence rate in the following manner:

$$
e=\log _{2}\left(\frac{\left|u_{j}-u_{j-1}\right|_{\mathcal{K}_{1}^{1}}}{\left|u_{j+1}-u_{j}\right|_{\mathcal{K}_{1}^{1}}}\right) .
$$

The quantity $e$ is not an exact convergence rate, but it turns out to be a quite reasonable approximation to it. Recall $h$ stands for the triangle size in $\left(\frac{1}{2}, \frac{3}{4}\right) \times(0, l)$. We have already seen that the correctly graded refinement improves the convergence rate with a factor of about $h^{0.43}$. In fact, the improvement may be better as our theory shows that the convergence rate in the case $\kappa<0.3$ is $h^{1}$ by Theorem 4.4.

Our theoretical prediction for the convergence rate in the case $\kappa=0.5$ is about $h^{0.59}$, and the theoretical prediction for the convergence rate in the cases $\kappa=0.1$, $\kappa=0.2$ is $h^{1}$, which is verified by Table 1. Moreover, one can see a big jump in the rates between $\kappa=0.25$ and $\kappa=0.35$, which strongly supports our theory for the critical number $\kappa \approx 0.3$. Thus, our numerical results completely agree with the theory we have presented in this paper. Based on the behavior of the sequence of the numbers in every column, it is also reasonable to expect the optimal rate by doing more refinements for all $\kappa<0.3$. Therefore, we conclude from our numerical results, that, for a correct refinement, the difference between consecutive numerical solutions is decreasing like $\operatorname{dim}\left(V_{n}\right)^{-1 / 2}$, which verifies Theorem 4.7 .

One may also notice that those numbers in each column keep increasing when $\kappa>0.3$. An explanation is that the solution consists of a singular and a regular part: $u=u_{s}+u_{r}$. The regular part $u_{r}$ dominates the behavior of the solution until $x$ is sufficiently close to the degenerate boundary, when the singular part can be taken into account [6]. Therefore, as shown in the table, the increasing rate slows down and will be fixed at some point.

\section{ACKNowledgments}

The author is grateful to Anna Mazzucato, Victor Nistor, Jinchao Xu, and Ludmil Zikatanov for helpful discussions and suggestions during this research.

\section{REFERENCES}

[1] R. Adams, Sobolev spaces, Pure and Applied Mathematics, vol. 65, Academic Press, New York, London, 1975. MR0450957 (56:9247)

[2] B. Ammann, A. D. Ionescu, and V. Nistor, Sobolev spaces and regularity for polyhedral domains, Documenta Mathematica 11 (2006), no. 2, 161-206. MR2262931

[3] B. Ammann and V. Nistor, Weighted sobolev spaces and regularity for polyhedral domains, Preprint, 2005. MR2339991(2008e:35028)

[4] T. Apel, Anisotropic finite elements: Local estimates and applications, Advances in Numerical Mathematics, B. G. Teubner, Stuttgart, 1999. MR.1716824(2000k:65002)

[5] T. Apel, S. Nicaise, and J. Schöberl, Finite element methods with anisotropic meshes near edges, Finite element methods (Jyväskylä, 2000), GAKUTO Internat. Ser. Math. Sci. Appl., vol. 15, Gakkōtosho, Tokyo, 2001, pp. 1-8. MR1896262

[6] T. Apel, A. Sändig, and J. R. Whiteman, Graded mesh refinement and error estimates for finite element solutions of elliptic boundary value problems in non-smooth domains, Math. Methods Appl. Sci. 19 (1996), no. 1, 63-85. MR1365264(96h:65144)

[7] D. N. Arnold, L. R. Scott, and M. Vogelius, Regular inversion of the divergence operator with Dirichlet boundary conditions on a polygon, Ann. Scuola Norm. Sup. Pisa Cl. Sci. (4) 15 (1988), no. 2, 169-192 (1989). MR 1007396 (91i:35043)

[8] I. Babuška, Finite element method for domains with corners, Computing (Arch. Elektron. Rechnen) 6 (1970), 264-273. MR0293858 (45:2934) 
[9] I. Babuška and A. K. Aziz, The mathematical foundations of the finite element method with applications to partial differential equations, Academic Press, New York, 1972. MR 0347104 $(49: 11824)$

[10] - On the angle condition in the finite element method, SIAM J. Numer. Anal. 13 (1976), no. 2, 214-226. MR0455462 (56:13700)

[11] I. Babuška, R. B. Kellogg, and J. Pitkäranta, Direct and inverse error estimates for finite elements with mesh refinements, Numer. Math. 33 (1979), no. 4, 447-471. MR553353 (81c:65054)

[12] I. Babuška and V. Nistor, Interior numerical approximation of boundary value problems with a distributional data, Numer. Methods Partial Differential Equations 22 (2006), no. 1, 79-113. MR2185526

[13] C. Bacuta and J. H. Bramble, Regularity estimates for solutions of the equations of linear elasticity in convex plane polygonal domains, Z. Angew. Math. Phys. 54 (2003), no. 5, 874878, Special issue dedicated to Lawrence E. Payne. MR2019187 (2005d:35049)

[14] C. Băcuţă, V. Nistor, and L. T. Zikatanov, Regularity and well posedness for the Laplace operator on polyhedral domains, IMA Preprint, 2004.

[15] _ Improving the rate of convergence of 'high order finite elements' on polygons and domains with cusps, Numer. Math. 100 (2005), no. 2, 165-184. MR2135780 (2006d:65130)

[16] _ Improving the rate of convergence of high-order finite elements on polyhedra. I. A priori estimates, Numer. Funct. Anal. Optim. 26 (2005), no. 6, 613-639. MR2187917 (2006i:35036)

[17] K. K. Boŭmatov, Estimates for solutions of strongly degenerate elliptic equations in weighted Sobolev spaces, Tr. Semin. im. I. G. Petrovskogo (2001), 194-239, 341. MR/1875964 (2003i:35112)

[18] J. H. Bramble and X. Zhang, Uniform convergence of the multigrid V-cycle for an anisotropic problem, Math. Comp. 70 (2001), no. 234, 453-470. MR1709148 (2001g:65134)

[19] S. Brenner and L. R. Scott, The mathematical theory of finite element methods, Texts in Applied Mathematics, vol. 15, Springer-Verlag, New York, 1994. MR:1278258 (95f:65001)

[20] Z. Cai and S. Kim, A finite element method using singular functions for the Poisson equation: corner singularities, SIAM J. Numer. Anal. 39 (2001), no. 1, 286-299 (electronic). MR1860726 (2002h:65177)

[21] P. Ciarlet, The finite element method for elliptic problems, Studies in Mathematics and Its Applications, vol. 4, North-Holland, Amsterdam, 1978. MR0520174 (58:25001)

[22] M. Costabel, Boundary integral operators on curved polygons, Ann. Mat. Pura Appl. (4) 133 (1983), 305-326. MR725031 (85m:44001)

[23] M. Dauge, Elliptic boundary value problems on corner domains, Lecture Notes in Mathematics, vol. 1341, Springer-Verlag, Berlin, 1988. MR961439 (91a:35078)

[24] R. DeVore and S. Dahlke, Besov regularity for elliptic boundary value problems, vol. PDE 22, Comm., 1997. MR1434135 (97k:35047)

[25] R. DeVore and G. G. Lorentz, Constructive approximation, Springer-Verlag, New York, 1993. MR1261635 (95f:41001)

[26] L. Evans, Partial differential equations, Graduate Studies in Mathematics, vol. 19, AMS, Rhode Island, 1998. MR 1625845 (99e:35001)

[27] V. Felli and M. Schneider, A note on regularity of solutions to degenerate elliptic equations of Caffarelli-Kohn-Nirenberg type, Adv. Nonlinear Stud. 3 (2003), no. 4, 431-443. MR2017240 (2004k:35151)

[28] D. A. French, The finite element method for a degenerate elliptic equation, SIAM J. Numer. Anal. 24 (1987), no. 4, 788-815. MR899704(88k:65110)

[29] J. Gopalakrishnan and J. E. Pasciak, The convergence of V-cycle multigrid algorithms for axisymmetric Laplace and Maxwell equations, Math. Comp. 75 (2006), no. 256, 1697-1719 (electronic). MR2240631 (2007g:65116)

[30] P. Grisvard, Elliptic problems in nonsmooth domains, Pitman Publishing, Massachusetts, 1985. MR775683(86m:35044)

[31] _ Singularities in boundary value problems, Research Notes in Applied Mathematics, vol. 22, Springer-Verlag, New York, 1992. MR 1173209 (93h:35004)

[32] W. B. Gu, C. Y. Wang, and B. Y. Liaw, Micro-macroscopic coupled modeling of batteries and fuel cells: Part 2. application to nickel-cadmium and nickel-metal hybrid cells, J. Electrochem. Soc. (1998). 
[33] R. B. Kellogg, Notes on piecewise smooth elliptic boundary value problems, 2003.

[34] V. A. Kondratiev, Boundary value problems for elliptic equations in domains with conical or angular points, Transl. Moscow Math. Soc. 16 (1967), 227-313. MR0226187 (37:1777)

[35] V. A. Kozlov, V. Mazya, and J. Rossmann, Elliptic boundary value problems in domains with point singularities, AMS, Rhode Island, 1997. MR.1469972 (98f:35038)

[36] _ Spectral problems associated with corner singularities of solutions of elliptic equations, Mathematical Surveys and Monographs, vol. 5, AMS, Rhode Island, 2001. MR1788991 (2001i:35069)

[37] M. Langlais, On the continuous solutions of a degenerate elliptic equation, Proc. London Math. Soc. (3) 50 (1985), no. 2, 282-298. MR.772714(86g:35082)

[38] J. E. Lewis and C. Parenti, Pseudodifferential operators of Mellin type, Comm. Partial Differential Equations 8 (1983), no. 5, 477-544. MR695401 (86f:35185)

[39] H. Li, A. Mazzucato, and V. Nistor, Analysis of the finite element method for transmission/ mixed boundary value problems on general polygonal domains, submitted (2008).

[40] H. Li and V. Nistor, Analysis of a modified Schrödinger operator in 2D: regularity, index, and FEM, J. Comput. Appl. Math., 2008, DOI:10.1016/j.cam.2008.05.009.

[41] J. M. Melenk and I. Babuška, The partition of unity finite element method: basic theory and applications, Comput. Methods Appl. Mech. Engrg. 139 (1996), no. 1-4, $289-314$. MR $1426012(97 \mathrm{k}: 65258)$

[42] S. A. Nazarov and B. A. Plamenevsky, Elliptic problems in domains with piecewise smooth boundaries, Expositions in Mathematics, vol. 13, de Gruyter, New York, 1994. MR1283387 (95h:35001)

[43] S. Rippa, Long and thin triangles can be good for linear interpolation, SIAM J. Numer. Anal. 29 (1992), no. 1, 257-270. MR1149097 (92j:65007)

[44] A. H. Schatz and L. B. Wahlbin, Maximum norm estimates in the finite element method on plane polygonal domains. I, Math. Comp. 32 (1978), no. 141, 73-109. MR0502065 $(58: 19233 \mathrm{a})$

[45] - Maximum norm estimates in the finite element method on plane polygonal domains. II. Refinements, Math. Comp. 33 (1979), no. 146, 465-492. MR0502067 (58:19233b)

[46] M. Taylor, Partial differential equations 1, basic theory, Applied Mathematical Sciences, vol. 115, Springer-Verlag, New York, 1995. MR1395147 (98b:35002a)

[47] L. B. Wahlbin, On the sharpness of certain local estimates for $\stackrel{\circ}{H^{1}}$ projections into finite element spaces: influence of a re-entrant corner, Math. Comp. 42 (1984), no. 165, 1-8. MR725981 (86b:65129)

[48] C. Y. Wang, W. B. Gu, and B. Y. Liaw, Micro-macroscopic coupled modeling of batteries and fuel cells: Part 1. model development, J. Electrochem. Soc. (1998).

[49] J. Xu, Iterative methods by space decomposition and subspace correction, SIAM Rev. 34 (1992), no. 4, 581-613. MR1193013 (93k:65029)

[50] K. Yosida, Functional analysis, fifth ed., A Series of Comprehensive Studies in Mathematics, vol. 123, Springer-Verlag, New York, 1978. MR0500055 (58:17765)

Department of Mathematics, Pennsylvania State University, University Park, PennSYLVANIA 16802

Current address: Department of Mathematics, Syracuse University, Syracuse, New York 13244

E-mail address: 1i_h@math.psu.edu 